\title{
Many-body effects in doped graphene on a piezoelectric substrate
}

DOI:

10.1103/PhysRevB.96.125119

\section{Document Version}

Final published version

Link to publication record in Manchester Research Explorer

\section{Citation for published version (APA):}

González, D. G., Zapata, I., Schiefele, J., Sols, F., \& Guinea, F. (2017). Many-body effects in doped graphene on a piezoelectric substrate. Physical Review B, 96(12), [125119]. https://doi.org/10.1103/PhysRevB.96.125119

\section{Published in:}

Physical Review B

\section{Citing this paper}

Please note that where the full-text provided on Manchester Research Explorer is the Author Accepted Manuscript or Proof version this may differ from the final Published version. If citing, it is advised that you check and use the publisher's definitive version.

\section{General rights}

Copyright and moral rights for the publications made accessible in the Research Explorer are retained by the authors and/or other copyright owners and it is a condition of accessing publications that users recognise and abide by the legal requirements associated with these rights.

\section{Takedown policy}

If you believe that this document breaches copyright please refer to the University of Manchester's Takedown Procedures [http://man.ac.uk/04Y6Bo] or contact uml.scholarlycommunications@manchester.ac.uk providing relevant details, so we can investigate your claim.

\section{OPEN ACCESS}




\title{
Many-body effects in doped graphene on a piezoelectric substrate
}

\author{
David G. González, ${ }^{1,2, *}$ Ivar Zapata, ${ }^{1}$ Jürgen Schiefele, ${ }^{1,3}$ Fernando Sols, ${ }^{1,2}$ and Francisco Guinea ${ }^{4,5}$ \\ ${ }^{1}$ Departamento de Física de Materiales, Universidad Complutense de Madrid, E-28040 Madrid, Spain \\ ${ }^{2}$ Campus de Excelencia Internacional, Campus Moncloa UCM-UPM, E-28040 Madrid, Spain \\ ${ }^{3}$ Instituto de Ciencia de Materiales de Madrid, CSIC, E-28 049 Madrid, Spain \\ ${ }^{4}$ IMDEA Nanociencia, Calle de Faraday 9, E-28 049 Madrid, Spain \\ ${ }^{5}$ Department of Physics and Astronomy, University of Manchester, Oxford Road, Manchester M13 9PL, United Kingdom
}

(Received 27 March 2017; revised manuscript received 16 August 2017; published 12 September 2017)

\begin{abstract}
We investigate the many-body properties of graphene on top of a piezoelectric substrate, focusing on the interaction between graphene electrons and piezoelectric acoustic phonons. We calculate the electron and phonon self-energies as well as the electron mobility limited by the substrate phonons. We emphasize the importance of proper screening of the electron-phonon vertex, and we discuss the various limiting behaviors as a function of electron energy, temperature, and doping level. The effect of piezoelectric acoustic phonons on graphene electrons is compared with that of intrinsic deformation acoustic phonons. Substrate phonons tend to dominate over intrinsic ones for low doping levels at high and low temperatures.
\end{abstract}

DOI: 10.1103/PhysRevB.96.125119

\section{INTRODUCTION}

Elastic waves supported by the boundaries of solids and, in particular, surface acoustic waves (SAWs) underlie numerous applications of microwave devices for signal processing [1]. SAWs with amplitudes of a few nanometers can be electrically excited on the surface of piezoelectric materials, and the resulting periodic deformation in adjacent thin film materials or quantum-well structures can be employed to modulate optical resonances in polaritonic or plasmonic devices [2-4]. Apart from the mechanical deformation, the vibration of the ionic lattice in a piezoelectric material produces an electric field traveling along with the SAW, which can transport charge carriers in monolayer graphene deposited on top of the piezomaterial [5-7] and, for instance, probe graphene's Landau level structure when an external magnetic field is applied [8].

Because the carbon allotrope graphene is an atomically thin all-surface material [9], its charge-carrier dynamics is very sensitive to the surrounding electromagnetic fields, and the possibility of changing graphene's carrier concentration in situ by applying an external gate voltage is a key feature in many graphene-based devices [10]. Ballistic charge transport in suspended graphene over micrometer distances and unprecedented carrier mobilities [11] are enabled by the high frequencies of the optical phonons in the stiff honeycomb lattice. Thus, the effects of electron-phonon scattering on transport are small in comparison with conventional metals [9]. However, in most device architectures, graphene is deposited on a substrate, and all lattice modes of the substrate material that induce an electric field will influence the carriers in the graphene sheet, making the choice of substrate material crucial for the resulting transport characteristics of the device [12]. This mechanism of remote phonon scattering in graphene has been mainly studied for substrates supporting optical phonon modes [13-21].

In the present work, we aim to clarify the role of acoustic piezoelectric surface phonons, which form the microscopic quanta of SAWs [22], in graphene-on-piezomaterial structures. After analyzing within a diagrammatic framework the effective carrier interaction due to an exchange of surface phonons in Sec. II, we study the self-energies acquired by both phonons and charge carriers in Secs. III and IV. While the renormalization of the Fermi velocity due to piezoelectric substrate phonons turns out to be small, we show that there are regimes where the substrate effects dominate the momentum relaxation mechanism in graphene. We compare both lifetimes and mobilities with the results obtained when only intrinsic acoustic deformation phonons are considered. The numerical results for mean free paths and electron mobilities shown in Sec. V are applicable to a variety of piezoelectric materials with different lattice structures and piezoelectric strengths.

Our study can be relevant for graphene devices operating in the ballistic transport regime, such as hot electron transistor devices [23] or field-effect transistors based on graphene on different piezoelectrics [24,25], and for scenarios in which quantum interference induces localization phenomena [26].

\section{EFFECTIVE ELECTRON-ELECTRON INTERACTION}

\section{A. Piezoelectric substrate}

The sound velocities $v_{s}(\theta)$ of piezoelectric acoustic phonons are anisotropic (depending on the direction angle $\theta$ ) and typically two or three orders of magnitude smaller than the Fermi velocity $v_{F}$ in graphene, which yields a relatively low value of the maximum acoustic frequency. Thus the dielectric screening effects due to the substrate can be described by its static (also anisotropic) dielectric constant $\varepsilon_{0}(\theta)$. This constant combines both core excitons and optical phonons as well as any high-frequency (instantaneous) polarization forces that screen the fields created by the piezoelectric acoustic phonons [27]. The Fourier transform of the repulsive Coulomb interaction thus reads

\footnotetext{
*d.gonzalez@mat.ucm.es
}

$$
v_{\mathbf{q}}^{(0)}=\frac{2 \pi e^{2}}{\bar{\varepsilon}_{0}(\theta) q},
$$


where $\bar{\varepsilon}_{0}(\theta)=\frac{\varepsilon_{0}(\theta)+1}{2}$ is the effective dielectric constant at the substrate-air interface [28], and $q=|\mathbf{q}|$ with $\mathbf{q}=\left(q_{x}, q_{y}\right)$ and $\theta \equiv \arg \left(q_{x}+i q_{y}\right)$.

The interaction between the graphene electrons and the piezoelectric acoustic (PA) phonons is given by

$$
H_{e-\mathrm{ph}}^{\mathrm{PA}}=\frac{1}{\sqrt{A}} \sum_{\mathbf{k}, \mathbf{q}, \sigma} \gamma_{\mathbf{q}}^{\mathrm{PA}} a_{\mathbf{k}+\mathbf{q}, \sigma}^{\dagger} a_{\mathbf{k}, \sigma} b_{\mathbf{q}}+\text { H.c. }
$$

Here, $A$ is the sample area, and $a_{\mathbf{k}, \sigma}$ is the Fermi operator for an electron of wave vector $\hbar \mathbf{k}$, spin-valley-cone index $\sigma$, and energy

$$
E_{k \sigma}=\hbar s v_{F} k,
$$

where $s= \pm 1$ is the cone index; $b_{\mathbf{q}}$ is the Bose operator for a substrate PA phonon of wave vector $\mathbf{q}$ and (directiondependent) frequency

$$
\omega_{\mathbf{q}}=v_{s}(\theta) q .
$$

As in Ref. [29], where a general form of the piezoelectric acoustic-phonon-electron interaction was derived (and compared to the general Fröhlich interaction with optical phonons), we assume that we only have to deal with phonons of momentum much smaller than the piezoelectric inverse lattice spacing (elastic limit). The electron-phonon coupling is then characterized by the $q$-independent vertex

$$
\gamma_{\mathbf{q}}^{\mathrm{PA}}=K_{R}(\theta)\left[\frac{\pi \alpha_{\mathrm{fs}} \hbar^{2} v_{F} v_{s}(\theta)}{\bar{\varepsilon}_{0}(\theta)}\right]^{\frac{1}{2}},
$$

where $\alpha_{\mathrm{fs}}=e^{2} /\left(\hbar v_{F}\right) \simeq 2.2$. It should be understood that, in the absence of the substrate, we are in the usual Fermi liquid regime of (doped) graphene. Quite generally, the electromechanical coupling coefficient satisfies $K_{R}(\theta)<1$. The general derivation of Eq. (5) is discussed in Ref. [29].

The coupling Eq. (5) enables a phonon-mediated electronelectron interaction

$$
V_{\mathrm{ph}}^{\mathrm{PA}}(\mathbf{q}, \omega)=\left|\gamma_{\mathbf{q}}^{\mathrm{PA}}\right|^{2} G_{0}^{\mathrm{PA}}(\mathbf{q}, \omega),
$$

where

$$
G_{0}^{\mathrm{PA}}(\mathbf{q}, \omega)=\frac{2 \omega_{\mathbf{q}} / \hbar}{\omega^{2}-\omega_{\mathbf{q}}^{2}+i 0^{+}}
$$

denotes the bare propagator of the surface acoustic phonons. By including screening effects due to the charge carriers in graphene, as described by the polarization $\Pi_{0}(q, \omega)$ (see Refs. [30,31]), we can define the total effective electronelectron interaction in terms of an anisotropic dielectric function $\varepsilon(\mathbf{q}, \omega)$ :

$$
\begin{aligned}
V_{\mathrm{eff}}(\mathbf{q}, \omega) & =\frac{2 \pi e^{2}}{\varepsilon(\mathbf{q}, \omega) q} \\
& =\frac{v_{\mathbf{q}}^{(0)}+V_{\mathrm{ph}}^{\mathrm{PA}}(\mathbf{q}, \omega)}{1-\left[v_{\mathbf{q}}^{(0)}+V_{\mathrm{ph}}^{\mathrm{PA}}(\mathbf{q}, \omega)\right] \Pi_{0}(q, \omega)} .
\end{aligned}
$$

Here and in the following, we adopt the convention of referring to $q$ as a subindex or argument when the dependence on $\mathbf{q}$ has circular symmetry. For low enough frequencies, typically $\hbar \omega \ll k_{B} T_{\mathrm{BG}}$, where $T_{\mathrm{BG}}$ is the Bloch-Grüneisen temperature [defined in Eq. (40)], the phonon-induced electron-electron interaction adopts a $q$ dependence similar to that of the Coulomb interaction:

$$
V_{\mathrm{ph}}^{\mathrm{PA}}(\mathbf{q}, \omega) \simeq-\frac{2\left|\gamma_{\mathbf{q}}^{\mathrm{PA}}\right|^{2}}{\hbar v_{s} q}
$$

By defining

$$
\varepsilon_{\mathrm{RPA}}(\mathbf{q}, \omega)=1-v_{\mathbf{q}}^{(0)} \Pi_{0}(q, \omega),
$$

we obtain for $\omega \rightarrow 0$

$$
\varepsilon_{\mathrm{RPA}}(\mathbf{q}, \omega) \simeq \varepsilon_{\mathrm{RPA}}(\mathbf{q}, 0),
$$

where the static dielectric function satisfies

$$
\varepsilon_{\mathrm{RPA}}(\mathbf{q}, 0)=1+\frac{k_{\mathrm{TF}}(\theta)}{q}
$$

for $q<2 k_{F}$, where

$$
k_{\mathrm{TF}}=\frac{4 \alpha_{\mathrm{fs}} k_{F}}{\bar{\varepsilon}_{0}(\theta)}
$$

is the (anisotropic) Thomas-Fermi wave vector and $k_{F}$ is the Fermi wave vector, the factor of 4 accounting for spin-valley degeneracy.

We may also define the renormalized phonon propagator

$$
\tilde{G}^{\mathrm{PA}}(\mathbf{q}, \omega)=\frac{G_{0}^{\mathrm{PA}}(\mathbf{q}, \omega)}{1-\frac{V_{\mathrm{ph}}^{\mathrm{PA}}(\mathbf{q}, \omega) \Pi_{0}(q, \omega)}{\varepsilon_{\mathrm{RPA}}(\mathbf{q}, \omega)}} .
$$

Then, Eq. (8) can be decomposed into an electron-electron and an electron-phonon part [27,32]. We obtain [29]

$$
\begin{aligned}
& V_{\mathrm{eff}}(\mathbf{q}, \omega)=\frac{v_{\mathbf{q}}^{(0)}}{\varepsilon_{\mathrm{RPA}}(\mathbf{q}, \omega)}+\left|\frac{\gamma_{\mathbf{q}}^{\mathrm{PA}}}{\varepsilon_{\mathrm{RPA}}(\mathbf{q}, \omega)}\right|^{2} \tilde{G}^{\mathrm{PA}}(\mathbf{q}, \omega), \\
& V_{\mathrm{eff}}(\mathbf{q}, \omega) \\
& V_{\mathbf{q}}^{\mathrm{RPA}}(\mathbf{q}, \omega)
\end{aligned}
$$

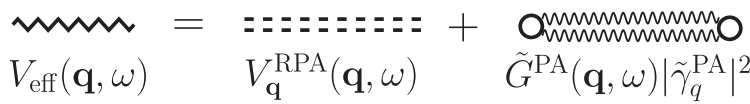

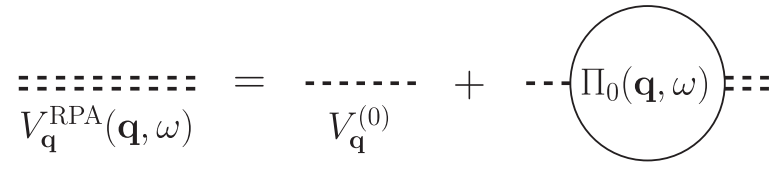
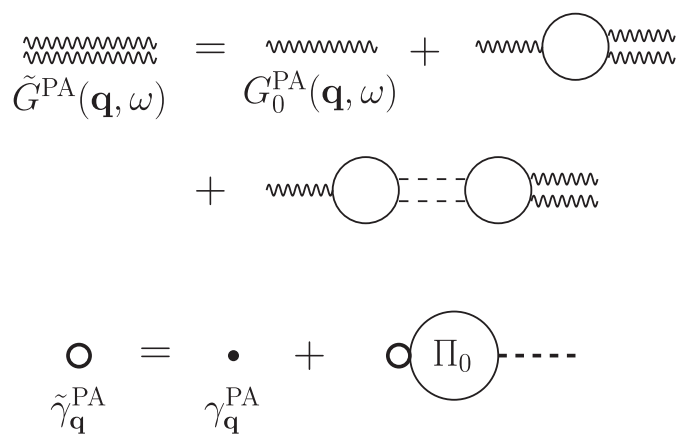

FIG. 1. Equivalent RPA scheme for the effective electronelectron interaction separated into an electron-electron Coulombic part and an electron-phonon part with a screened vertex and renormalized phonon propagator. In the second line, $V^{\mathrm{RPA}}(\mathbf{q}, \omega)$ is the first term of Eq. (15) 
TABLE I. Angle-averaged values of the electromechanical coupling coefficient $K_{R}^{2}$ appearing in Eq. (5), the effective dielectric constant $\bar{\varepsilon}_{0}$, the sound velocity $v_{s}$, the vertex strength $\gamma_{\mathbf{q}}^{\mathrm{PA}}$ [see Eq. (5)], the dimensionless coupling strength $\lambda_{e \text {-ph }}$ defined in Eq. (17), and the ratio $k_{\mathrm{TF}} / k_{F}$ for several materials. Numerical values of the elastic, piezoelectric, and dielectric tensors have been taken from Refs. [33-35] and references therein.

\begin{tabular}{lcccccc}
\hline \hline Material & Cut & $K_{R}^{2}$ & $\bar{\varepsilon}_{0}$ & $v_{s}\left(\frac{\mathrm{cm}}{\mathrm{s}}\right)$ & $\left|\gamma_{\mathbf{q}}^{\mathrm{PA}}\right|^{2}(\mathrm{eV} \mathrm{cm})^{2}$ & $\lambda_{e-\mathrm{ph}}$ \\
\hline $\mathrm{GaAs}($ cubic) & $X-Y-Z$ & 0.0015 & 6.9 & $2.70 \times 10^{5}$ & $1.71 \times 10^{-20}$ & 0.0019 \\
$\mathrm{ZnO}(6 \mathrm{~mm})$ & $Z$ cut & 0.016 & 4.8 & $2.71 \times 10^{5}$ & $2.70 \times 10^{-19}$ & 0.029 \\
$\mathrm{ZnO}(6 \mathrm{~mm})$ & $X$ cut & 0.0064 & 4.8 & $2.63 \times 10^{5}$ & $6.60 \times 10^{-20}$ & 0.0074 \\
$\mathrm{AlN}(6 \mathrm{~mm})$ & $Z$ cut & 0.0026 & 5.0 & $5.85 \times 10^{5}$ & $9.18 \times 10^{-20}$ & 0.0046 \\
$\mathrm{AlN}(6 \mathrm{~mm})$ & $X$ cut & 0.0048 & 5.0 & $5.81 \times 10^{5}$ & $1.66 \times 10^{-19}$ & 0.0084 \\
$\mathrm{LiNbO}_{3}(3 \mathrm{~m})$ & $Z$ cut & 0.0068 & 19 & $3.85 \times 10^{5}$ & $4.25 \times 10^{-20}$ & 0.0032 \\
$\mathrm{LiNbO}_{3}(3 \mathrm{~m})$ & $Y$ cut & 0.017 & 20 & $3.59 \times 10^{5}$ & $9.35 \times 10^{-20}$ & 0.0077 \\
$\mathrm{LiNbO}_{3}(3 \mathrm{~m})$ & $X$ cut & 0.019 & 20 & $3.60 \times 10^{5}$ & $9.80 \times 10^{-20}$ & 0.0080 \\
$\mathrm{PZT}^{-2}(6 \mathrm{~mm})$ & $Z$ cut & 0.027 & 350 & $2.26 \times 10^{5}$ & $5.37 \times 10^{-21}$ & $7.0 \times 10^{-4}$ \\
$\mathrm{PZT}-4(6 \mathrm{~mm})$ & $X$ cut & 0.0021 & 350 & $1.80 \times 10^{5}$ & $3.17 \times 10^{-22}$ & 0.44 \\
\hline \hline
\end{tabular}

as shown diagrammatically in Fig. 1. We wish to emphasize the importance of electronic screening of the electron-phonon vertex shown in Eq. (15). This will strongly influence the role of scattering processes involving low values of $q$.

\section{B. Input parameters and main approximations}

The input parameters of our study are those that characterize the piezoelectric substrate, namely $K_{R}(\theta), v_{s}(\theta)$, and $\bar{\varepsilon}_{0}(\theta)$, from which the electron-phonon interaction $\gamma_{\mathbf{q}}^{\mathrm{PA}}(\theta)$ is computed [see Eq. (5)]. On the other hand, the graphene electron system is characterized by its doping level as given by $k_{F}$. A dimensionless parameter $\lambda_{e \text {-ph }}(\theta)$ characterizing the strength of the coupling of Eqs. (5) and (9) can be obtained from multiplying the resulting effective interaction (6) at $q=k_{F}$ by the density of states at the Fermi energy,

$$
D\left(E_{F}\right)=-\Pi_{0}\left(k_{F}, 0\right)=\frac{2 k_{F}}{\pi \hbar v_{F}},
$$

which leads to

$$
\begin{aligned}
\lambda_{e-\mathrm{ph}}(\theta) & \equiv V_{\mathrm{ph}}^{\mathrm{PA}}\left(k_{F} \hat{\mathbf{q}}, 0\right) \Pi_{0}\left(k_{F}, 0\right) \\
& =\frac{4}{\pi \hbar^{2} v_{s} v_{F}}\left|\gamma_{\mathbf{q}}^{\mathrm{PA}}\right|^{2}=4 K_{R}^{2}(\theta) r_{s}(\theta),
\end{aligned}
$$

where $\hat{\mathbf{q}}=\mathbf{q} / q$ and the parameter

$$
r_{s}(\theta) \equiv \frac{\alpha_{\mathrm{fs}}}{\bar{\varepsilon}_{0}(\theta)}
$$

characterizes the ratio between the interaction and kinetic energies. This yields for the ratio between the piezoelectric interaction and the residual static Coulomb repulsion [29]

$$
\frac{\lambda_{e-\mathrm{ph}}}{\lambda_{e-e}}=K_{R}^{2},
$$

where

$$
\lambda_{e-e}(\theta)=v_{k_{F} \hat{\mathbf{q}}}^{(0)} \Pi\left(k_{F}, 0\right)=\frac{4 \alpha_{\mathrm{fs}}}{\bar{\varepsilon}_{0}(\theta)}=4 r_{s}(\theta)
$$

is the dimensionless electron-electron coupling strength in substrate-screened graphene.

The electromechanical coupling coefficient $K_{R}(\theta)$, characteristic of each piezoelectric material, can be measured in
SAW experiments. It depends on the material's piezoelectric, elastic, and dielectric tensors, as well as on its mass density. In Table I we summarize angle-averaged values for selected representative materials as computed from the data given in Refs. [33-35].

For example, the materials considered in Ref. [4], namely $\mathrm{ZnO}$ and $\mathrm{AlN}$, have associated piezoelectric tensors that are much larger than those of GaAs [36], which increases the electron-phonon coupling by more than one order of magnitude. But there exist piezoelectric materials whose coefficients are even larger, such as, e.g., $\mathrm{LiNbO}_{3}, \mathrm{BaTiO}_{3}$, or the PZT (lead zirconate titanate) $\mathrm{PbTi}_{x} \mathrm{Zr}_{1-x} \mathrm{O}_{3}$, among many oxides with the perovskite structure and formula $A B \mathrm{O}_{3}$, which tend to show ferroelectric properties, and they are sometimes reminiscent of the layers between $\mathrm{CuO}_{2}$ planes in cuprate high-temperature superconductors. Despite being more piezoelectric, the dielectric tensors in these ferroelectrics are so high that the interaction decreases [but not the ratio to the also highly screened Coulomb repulsion; see Eq. (19)]. We recall in this regard that, roughly, $K_{R}^{2} \sim \hat{e}^{2} /\left(\hat{c} \bar{\varepsilon}_{0}\right)$, where $\hat{e}$ is a value for the piezoelectric tensor and $\hat{c}$ for the elastic tensor [37].

The point group of $\mathrm{ZnO}$ and $\mathrm{AlN}$ gives isotropic couplings with the $Z$ cut and therefore isotropic sound velocities. On the other hand, their $X$ and $Y$ cuts are equivalent. This does not happen, for example, in $\mathrm{LiNbO}_{3}$, whose $K_{R}^{2}(\theta)$ and vertex values in the $X, Y$, and $Z$ cuts are shown in Fig. 2 as an example. For some graphs of the velocities in different cuts, see, for example, Ref. [38]. Here the cut refers to the lattice axis perpendicular to the surface; for a detailed description of the cut terminology, see, e.g., Refs. [39,40].

In the previous subsection, we have introduced the approximation of assuming a flat interface and taking the elastic (long phonon wavelength) limit for the piezoelectric, as expressed in Eq. (5) and used in Ref. [29]. We will also make the common assumption that the interface is large enough to permit the neglect of geometrical effects due to finite-size boundaries. This is expected to be a good approximation for system sizes much larger than the length scales of the problem, namely $k_{F}^{-1}$ and $k_{\mathrm{TF}}^{-1}$. In the discussion of analytical limits, as well as in the tractable computation of scattering rates, we will often take angle-averaged substrate parameters. 

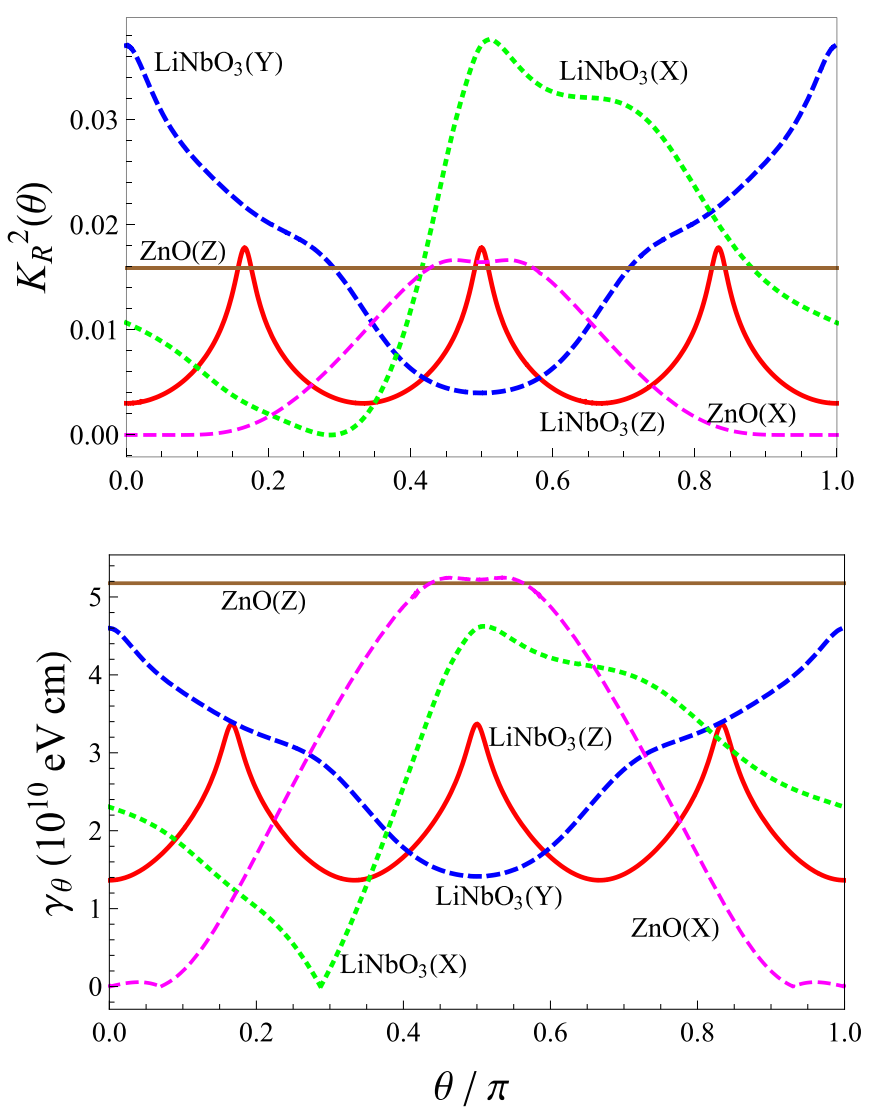

FIG. 2. Some representative magnitudes for SAW phonons and for two different materials with different symmetries $\left(\mathrm{LiNbO}_{3}\right.$ and $\mathrm{ZnO}$ ), as a function of the angle within the crystal plane parallel to the cut plane, in the $X-Y-Z$ cuts (denoted between parentheses). For the $Z$ cut ( $X$ cut $/ Y$ cut), the zero angle corresponds to the $x$ axis ( $y$ axis $/ z$ axis) with the angle growing toward the $y$ axis ( $z$ axis $/ x$ axis). See Refs. $[39,40]$ for a further account of the cut language convention. Upper: electromechanical coupling coefficient. Lower: electron-phonon vertex. The plots are obtained by processing data for the elastic, piezoelectric, and dielectric tensors taken from Refs. [33-35] and references therein.

\section{PHONON SELF-ENERGY}

As the piezoelectric coupling Eq. (2) enables the transfer of energy between carriers in graphene and the phonon modes of the substrate material, the latter acquire an extra decay rate due to Landau damping. To assess the magnitude of this effect, we proceed to estimate the self-energy of the substrate phonons due to their interactions with graphene carriers. Substituting the bare propagator (7) into (14), we obtain

$$
\tilde{G}^{\mathrm{PA}}(\mathbf{q}, \omega)=\frac{2 \omega_{\mathbf{q}} / \hbar}{\omega^{2}-\omega_{\mathbf{q}}^{2}-2 \hbar^{-1} \omega_{\mathbf{q}}\left|\gamma_{\mathbf{q}}^{\mathrm{PA}}\right|^{2} \frac{\Pi_{0}(q, \omega)}{\varepsilon_{\mathrm{RPA}}(\mathbf{q}, \omega)}} .
$$

In the phonon frequency range $\omega \sim v_{s} q \ll v_{F} q$ in which we will be mostly interested, we can approximate (see, e.g., Ref. [26])

$$
\Pi_{0}(q, \omega) \simeq-D\left(E_{F}\right)\left(1+i \frac{\omega}{v_{F} q}\right)
$$

in the RPA electron-electron dielectric function Eq. (10), so that, in terms of the parameter $\lambda_{e-\mathrm{ph}}(\theta)$, the poles of $\tilde{G}^{\mathrm{PA}}$ are shifted to

$$
\begin{aligned}
\tilde{\omega}_{\mathbf{q}}= & \pm v_{s} q\left(1-\lambda_{e-\mathrm{ph}} \frac{k_{F}}{q+k_{\mathrm{TF}}}\right)^{\frac{1}{2}} \\
& \mp i \lambda_{e-\mathrm{ph}} \frac{v_{s}}{v_{F}} \frac{v_{s} k_{F}}{2}\left(\frac{q}{q+k_{\mathrm{TF}}}\right)^{2} .
\end{aligned}
$$

In the long-wavelength limit $\left(q \ll k_{F}\right)$, the leading order of the ratio of the imaginary and real parts of the dressed phononic energy goes like

$$
\left|\frac{\operatorname{Im}\left(\tilde{\omega}_{\mathbf{q}}\right)}{\operatorname{Re}\left(\tilde{\omega}_{\mathbf{q}}\right)}\right| \simeq \frac{1}{2} K_{R}^{2} \frac{v_{s}}{v_{F}}\left(\frac{q}{k_{\mathrm{TF}}}\right)^{ \pm 1},
$$

where $\left(q / k_{\mathrm{TF}}\right)^{ \pm 1} \ll 1$, the case $k_{\mathrm{TF}} \ll q \ll k_{F}$ being meaningful only in those materials where $k_{\mathrm{TF}}$ is substantially smaller than $k_{F}$. Due to the fact that $v_{F} / v_{s} \sim 300$ and to the $K_{R}^{2}(\theta)$ values shown in Table I for typical materials, the lifetime of the phonons can be neglected in all analyzed regimes. It can also be shown that, near the quasiparticle poles, the residue $Z_{\mathbf{q}}$ is close to unity (i.e., the wave-function renormalization is weak):

$$
\begin{gathered}
\tilde{G}^{\mathrm{PA}}(\mathbf{q}, \omega) \simeq Z_{\mathbf{q}} \frac{2 \tilde{\omega}_{\mathbf{q}} / \hbar}{\omega^{2}-\tilde{\omega}_{\mathbf{q}}^{2}}, \\
Z_{\mathbf{q}} \simeq 1+\lambda_{\mathrm{e}-\mathrm{ph}} \frac{k_{F}}{q+k_{\mathrm{TF}}} .
\end{gathered}
$$

Thus in the following we can assume the substrate phonons to be well-defined, stable quasiparticles, and we will approximate the renormalized phonon propagator (25) by the bare one, Eq. (7).

\section{ELECTRON SELF-ENERGY}

\section{A. General expressions}

We focus on the case of $n$-doped graphene $\left(E_{F}>0\right)$ so that we will be interested in the electron self-energies at energies $\hbar \omega$ in the upper Dirac cone. With an effective electron-electron interaction $V_{\text {eff }}$ given in (15), the self-energy acquired by the charge carriers in graphene (within the $G_{0} W$ approximation, as indicated in Fig. 3) has the general form

$$
\begin{aligned}
\Sigma_{+}\left(\mathbf{k}, i \omega_{n}\right)= & -k_{B} T \sum_{s= \pm} \sum_{\mathbf{q}} \sum_{i v_{n}} F_{+s}(\mathbf{k}, \mathbf{k}+\mathbf{q}) \\
& \times G_{0, s}^{\mathrm{el}}\left(\mathbf{k}+\mathbf{q}, i \omega_{n}+i v_{n}\right) V_{\mathrm{eff}}\left(\mathbf{q}, i v_{n}\right),
\end{aligned}
$$

where the subscript + refers to the conduction band (the calculation for $\Sigma_{-}$being analogous), the index $s= \pm$ is summed over both bands,

$$
G_{0, s}^{\mathrm{el}}(\mathbf{k}, \omega)=\left(\omega-E_{\mathbf{k} s}-\mu\right)^{-1}
$$

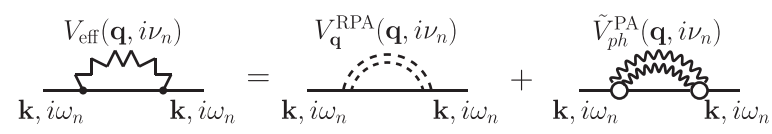

FIG. 3. Electron self-energy in the $G_{0} W$ approximation; see Eq. (27). 
denotes the (bare) electron propagator, $i v_{n}$ and $i \omega_{n}$ are, respectively, the bosonic and fermionic Matsubara frequencies, and the spinor overlap factor

$$
F_{+s}(\mathbf{k}, \mathbf{k}+\mathbf{q})=\frac{1}{2}(1+s \cos \alpha)
$$

arises due to the sublattice structure of graphene [9], $\alpha$ being the angle formed by $\mathbf{k}$ and $\mathbf{k}+\mathbf{q}$.

Expression (15) for $V_{\text {eff }}$ allows us to separate the self-energy $\Sigma_{+}$into contributions due to electron-electron and electronphonon interactions. While the former has been considered in Refs. [26,41], the contributions of graphene-intrinsic optical or acoustic phonons, as well as optical substrate phonons, to the electron self-energy have been studied in Refs. [21,26]. Thus in the present work we focus entirely on the effect of piezoelectric acoustic substrate phonons, as expressed in the self-energy

$$
\begin{aligned}
\Sigma_{+}^{\mathrm{PA}}\left(\mathbf{k}, i \omega_{n}\right)= & -k_{B} T \sum_{s= \pm} \sum_{\mathbf{q}} \sum_{i v_{n}} F_{+s}(\mathbf{k}, \mathbf{k}+\mathbf{q}) \\
& \times G_{0,+}^{\mathrm{el}}\left(\mathbf{k}+\mathbf{q}, i \omega_{n}+i v_{n}\right) \tilde{V}_{\mathrm{ph}}^{\mathrm{PA}}\left(\mathbf{q}, i v_{n}\right),
\end{aligned}
$$

where

$$
\tilde{V}_{\mathrm{ph}}^{\mathrm{PA}}(\mathbf{q}, \omega) \equiv\left|\frac{\gamma_{\mathbf{q}}^{\mathrm{PA}}}{\varepsilon_{\mathrm{RPA}}(\mathbf{q}, \omega)}\right|^{2} \tilde{G}^{\mathrm{PA}}(\mathbf{q}, \omega) .
$$

To sum over over Matsubara frequencies, we follow Ref. [27] and approximate the vertex renormalization by its static limit [see Eq. (12)] while neglecting the phonon self-energy, i.e., in Eq. (30) we replace $\tilde{V}_{\mathrm{ph}}^{\mathrm{PA}}(\mathbf{q}, \omega)$ by

$$
\bar{V}_{\mathrm{ph}}^{\mathrm{PA}}(\mathbf{q}, \omega) \equiv\left|\frac{\gamma_{\mathbf{q}}^{\mathrm{PA}}}{\varepsilon_{\mathrm{RPA}}(\mathbf{q}, 0)}\right|^{2} G_{0}^{\mathrm{PA}}(\mathbf{q}, \omega) .
$$

We arrive at the following retarded self-energy:

$$
\begin{aligned}
\Sigma_{+}^{\mathrm{PA}}(\mathbf{k}, \omega)= & \sum_{s= \pm} \int \frac{d \mathbf{q}}{(2 \pi)^{2}}\left|\frac{\gamma_{\mathbf{q}}^{\mathrm{PA}}}{\varepsilon_{\mathrm{RPA}}(\mathbf{q}, 0)}\right|^{2} F_{+s}\left(\mathbf{k}, \mathbf{k}^{\prime}\right) \\
& \times\left[\frac{n_{B}\left(\hbar \omega_{\mathbf{q}}\right)+n_{F}\left(\epsilon_{k^{\prime} s}\right)}{\hbar \omega+\hbar \omega_{\mathbf{q}}-\epsilon_{k^{\prime} s}+i 0^{+}}\right. \\
& \left.+\frac{n_{B}\left(\hbar \omega_{\mathbf{q}}\right)+1-n_{F}\left(\epsilon_{k^{\prime} s}\right)}{\hbar \omega-\hbar \omega_{\mathbf{q}}-\epsilon_{k^{\prime} s}+i 0^{+}}\right]
\end{aligned}
$$

where $\mathbf{k}^{\prime}$ stands for $\mathbf{k}^{\prime} \equiv \mathbf{k}+\mathbf{q}$,

$$
\begin{aligned}
n_{B}\left(\hbar \omega_{\mathbf{q}}\right) & =\left[\exp \left(\frac{\hbar \omega_{\mathbf{q}}}{k_{B} T}\right)-1\right]^{-1}, \\
n_{F}\left(\epsilon_{k^{\prime} s}\right) & =\left[\exp \left(\frac{\epsilon_{k^{\prime} s}}{k_{B} T}\right)+1\right]^{-1}
\end{aligned}
$$

denote the Bose and Fermi distributions, respectively, and the energies $\epsilon_{k s}=E_{k s}-\mu$ are taken relative to the chemical potential. We proceed by evaluating the real and imaginary parts of Eq. (33) separately. Hereafter, we assume $T \ll T_{F}$ so that the zero-temperature RPA dielectric function can be used [30]. Since $\mu \simeq E_{F}$, we can write

$$
\epsilon_{k s}=\hbar v_{F}\left(k s-k_{F}\right) \text {. }
$$

\section{B. Imaginary part}

The imaginary part of Eq. (33) acquires the form

$$
\begin{aligned}
\operatorname{Im} \Sigma_{+}^{\mathrm{PA}}(\mathbf{k}, \omega)= & -\pi \sum_{s= \pm} \sum_{t= \pm} \int \frac{d \mathbf{q}}{(2 \pi)^{2}}\left|\frac{\gamma_{\mathbf{q}}^{\mathrm{PA}}}{\varepsilon_{\mathrm{RPA}}(\mathbf{q}, 0)}\right|^{2} \frac{1+s \cos \alpha}{2} \\
& \times\left[n_{F}\left(\hbar \omega_{\mathbf{q}}+t \hbar \omega\right)+n_{B}\left(\hbar \omega_{\mathbf{q}}\right)\right] \\
& \times \delta\left(\hbar \omega+t \hbar \omega_{\mathbf{q}}-\epsilon_{k^{\prime} s}\right),
\end{aligned}
$$

where $t= \pm 1$ corresponds to the absorption or emission of a phonon, respectively.

Setting $\omega=\epsilon_{k+}$ in Eq. (37), that is, considering the on-shell self-energy, yields the value $\hbar /(2 \tau)$ for the decay width of charge carriers with wave vector $\mathbf{k}$. Here we are assuming that the renormalization of the Fermi energy $\Delta E_{F}=$ $\operatorname{Re} \Sigma^{\mathrm{PA}}\left(k_{F}, 0\right)$, as given by the pole of the dressed electron propagator, is tiny, as can be checked in the next section [see Eq. (58) and related ones]. To obtain analytical expressions for the asymptotic behaviors of the on-shell self-energy, we introduce the quasielastic approximation

$$
\delta\left(\epsilon_{k+}+t \hbar \omega_{\mathbf{q}}-\epsilon_{k^{\prime} s}\right) \simeq \delta\left(\epsilon_{k+}-\epsilon_{k^{\prime} s}\right)
$$

in Eq. (37), which is well justified since $v_{F} / v_{s} \sim 300$. As we are working with $k_{F}>0$, the $s=-$ term is null. Hereafter, $\epsilon_{k}$ will be equivalent to $\epsilon_{k+}$, so that

$$
\epsilon_{k}=\hbar v_{F}\left(k-k_{F}\right) .
$$

For magnitude estimates, we will assume $\epsilon_{k}>0$.

The relevant scale for finite-temperature effects in graphene, where carrier densities are much smaller than in conventional metals, is the Bloch-Grüneisen temperature $T_{\mathrm{BG}}$, defined as the scale of the acoustic phonons in the Fermi sea,

$$
k_{B} T_{\mathrm{BG}} \equiv 2 \hbar v_{s} k_{F} .
$$

\section{Zero temperature, small $k$}

Then at zero temperature (by which we mean $T \ll$ $\left.\epsilon_{k} / k_{B}, T_{\mathrm{BG}}\right), n_{F}$ in (37) becomes a step function that cuts off the momentum integration, while $n_{B}$ vanishes. Then, in the limit $\epsilon_{k} \ll \hbar v_{s} k_{\mathrm{TF}}$ [for which the largest contributing $q$ in (37) is $q \sim \epsilon_{k} / \hbar v_{s}$ so that we can assert $q \ll k_{\mathrm{TF}}$ ] the quasiparticle lifetime decays as $\epsilon_{k}^{3}$ near the Fermi surface while depending on the direction of the $\mathbf{k}$ vector:

$$
\begin{aligned}
-\operatorname{Im} \Sigma_{+}^{\mathrm{PA}}\left(\mathbf{k}, \epsilon_{k}\right) & \simeq \frac{1}{6 \pi} \frac{\left|\gamma_{\perp}^{\mathrm{PA}}\right|^{2}}{\hbar v_{F} k_{\mathrm{TF} \perp}^{2}} \frac{\epsilon_{k}^{3}}{\left(\hbar v_{s \perp}\right)^{3}} \\
& =\frac{\lambda \perp}{24}\left(\frac{k_{F}}{k_{\mathrm{TF} \perp}}\right)^{2}\left(\frac{v_{F}}{v_{s \perp}}\right)^{2}\left(\frac{\epsilon_{k}}{E_{F}}\right)^{3} \hbar v_{F} k_{F},
\end{aligned}
$$

where all the substrate-related constants, like $\lambda_{\perp} \equiv \lambda_{\text {e-ph }}\left(\theta_{\perp \mathbf{k}}\right)$ of Eq. (17), have to be taken in the direction $\theta_{\perp \mathbf{k}}$ perpendicular to $\mathbf{k}$. The fast $\epsilon_{k}^{3}$ decrease (as $\epsilon_{k} \rightarrow 0$ ) is due to the vertex renormalization, since $\varepsilon_{\mathrm{RPA}}$ in Eq. (37) diverges for $q \ll k_{\mathrm{TF}}$ [see Eq. (12)].

Hereafter we remove the subindex $\perp$ from the anisotropic parameters in those expressions where they are assumed to be angle-independent or only their order of magnitude matters. 


\section{Zero temperature, larger $k$}

For $\epsilon_{k} \gg \hbar v_{s} k_{\mathrm{TF}}$ we obtain the result

$$
\begin{aligned}
-\operatorname{Im} \Sigma_{+}^{\mathrm{PA}}\left(k, \epsilon_{k}\right) & \simeq \frac{\lambda}{4} \hbar v_{s} k \int_{0}^{1} \frac{y^{2} \sqrt{1-y^{2}}}{\left(y+\frac{k_{\mathrm{TF}}}{2 k}\right)^{2}} d y \\
& =\frac{\lambda \hbar v_{s} k}{4} f\left(\frac{k_{\mathrm{TF}}}{2 k}\right), \\
f(x)=3 x+\frac{\pi}{4}\left(1-6 x^{2}\right) & +\frac{\left(3 x^{3}-2 x\right) \operatorname{acosh}\left(x^{-1}\right)}{\sqrt{1-x^{2}}} .
\end{aligned}
$$

This admits two regimes: for $\hbar v_{s} k_{\mathrm{TF}} \ll \epsilon_{k} \ll E_{F}$,

$$
-\operatorname{Im} \Sigma_{+}^{\mathrm{PA}}\left(k, \epsilon_{k}\right) \simeq \frac{\lambda k_{B} T_{\mathrm{BG}}}{8} f\left(\frac{2 \alpha_{\mathrm{fs}}}{\bar{\varepsilon}_{0}}\right),
$$

while for $\epsilon_{k} \gg E_{F}$ we obtain

$$
-\operatorname{Im} \Sigma_{+}^{\mathrm{PA}}\left(k, \epsilon_{k}\right) \simeq \frac{\pi \lambda v_{s}}{16 v_{F}} \epsilon_{k} .
$$

Returning to the low-energy $\left(\epsilon_{k} \ll \hbar v_{s} k_{\mathrm{TF}}\right)$ regime [see Eq. (41)], we note that, without the vertex screening effect [that is, setting $\varepsilon_{\text {RPA }} \rightarrow 1$ in Eq. (37)], instead of the $\epsilon_{k}^{3}$ behavior one would find the linear $\epsilon_{k}$ dependence characteristic of a marginal Fermi liquid,

$$
-\operatorname{Im} \Sigma_{+(\text {no scr })}^{\mathrm{PA}}\left(\mathbf{k}, \epsilon_{k}\right) \simeq \frac{\lambda_{\perp}}{8} \epsilon_{k},
$$

which (for materials such that $k_{\mathrm{TF}} \ll k_{F}$ ) behaves similarly to the true self-energy in the range $\hbar v_{s} k_{\mathrm{TF}} \ll \epsilon_{k} \ll \hbar v_{s} k_{F}$, since $\varepsilon_{\mathrm{RPA}}$ tends to unity for the momenta $q \gg k_{\mathrm{TF}}$ dominating the integral (37). We will see, however, that a small offset remains due to the contribution of the screened low- $q$ values $\left(q \ll k_{\mathrm{TF}}\right)$.

Table I shows representative angle-independent material parameters, including those that will be used for the numerical calculations discussed in Sec. V. From Eqs. (41), (46), and the parameter values shown in Table I, it is safe to conclude that, at zero temperature, the damping rate due to electronphonon coupling is always much smaller than $\epsilon_{k}$. Thus the single-electron quasiparticles near the Fermi surface are well defined.

\section{Small $k$, low nonzero temperature}

So far we have assumed zero temperature, i.e., $k_{B} T \ll$ $\epsilon_{k}$. At nonzero temperatures, the vertex renormalization is fundamental to avoid logarithmic divergences. These occur for the unscreened self-energy at any nonzero temperature due to the divergent contribution of small- $q$ values. Focusing on the correctly screened self-energy, we consider first the nonzero, low-temperature limit $\epsilon_{k} \ll k_{B} T \ll 2 \hbar v_{s} k_{\mathrm{TF}}, k_{B} T_{\mathrm{BG}}$. Again, only the perpendicular-to-k substrate-related constants appear. We obtain

$$
-\operatorname{Im} \Sigma_{+}^{\mathrm{PA}}\left(k, \epsilon_{k}\right) \simeq \lambda_{\perp} k_{B} T\left(\frac{k_{F}}{k_{\mathrm{TF} \perp}}\right)^{2}\left(\frac{T}{T_{\mathrm{BG} \perp}}\right)^{2} \frac{7 \zeta(3)}{2},
$$

with $7 \zeta(3) / 2 \simeq 4.21$. The essential independence from $k$ of the lifetime (which allows for the replacement $k \simeq k_{F}$ ) is a general property of the case $\epsilon_{k} \ll T$. In those materials where $\varepsilon_{0}$ is so high that $k_{\mathrm{TF}} \ll k_{F}$ and therefore a temperature regime exists such that $\epsilon_{k} \ll \hbar v_{s} k_{\mathrm{TF}} \ll k_{B} T \ll k_{B} T_{\mathrm{BG}}$, the $T^{3}$ law is replaced by a $\sim T \log T$ behavior. Specifically, the asymptotic expression reads

$$
-\operatorname{Im} \Sigma_{+}^{\mathrm{PA}}\left(k, \epsilon_{k}\right) \simeq \lambda_{\perp} k_{B} T\left(\frac{k_{F}}{k_{\mathrm{TF} \perp}}\right)^{2} \log \left(\frac{\hbar v_{s} k_{\mathrm{TF}}}{k_{B} T}\right) .
$$

\section{Small $k$, high temperature}

The high-temperature limit $\left(T_{\mathrm{BG}} \ll T\right.$, while only $\epsilon_{k} \ll E_{F}$ is required), where phonons are nondegenerate, yields

$$
\begin{aligned}
-\operatorname{Im} \Sigma_{+}^{\mathrm{PA}}\left(k, \epsilon_{k}\right) & \simeq \frac{\lambda}{4} k_{B} T \int_{0}^{1} \frac{y \sqrt{1-y^{2}}}{\left(y+\frac{k_{\mathrm{TF}}}{2 k}\right)^{2}} d y \\
& =\frac{\lambda k_{B} T}{4} g\left(\frac{k_{\mathrm{TF}}}{2 k}\right), \\
g(x)=-2+\pi x & +\frac{\left(1-2 x^{2}\right) \operatorname{acosh}\left(x^{-1}\right)}{\sqrt{1-x^{2}}} .
\end{aligned}
$$

The logarithmic divergence of the function $g$ at $x \rightarrow 0$ becomes relevant in the limit $k \gg k_{\mathrm{TF}}$, where

$$
-\operatorname{Im} \Sigma_{+}^{\mathrm{PA}}\left(k, \epsilon_{k}\right) \simeq \frac{\lambda k_{B} T}{4}\left[\log \left(\frac{4 k}{k_{\mathrm{TF}}}\right)-2\right] .
$$

\section{Comparison with graphene intrinsic phonons}

Comparing Eqs. (41), (47), and (49) with the corresponding limiting expressions for the electron self-energy induced by the graphene-intrinsic deformation-potential acoustic (DA) phonons [26], we see below that, for an important range of parameter values, the inverse lifetime is dominated by the piezoelectric substrate phonons.

For our estimates we borrow $\Sigma_{+}^{\mathrm{DA}}\left(k, \epsilon_{k}\right)$ from Ref. [26]. Specifically, with a deformation constant $D \simeq 25 \mathrm{eV}$, and taking $k_{F}=\left[k_{F}\right] 10^{6} \mathrm{~cm}^{-1}$ (this momentum unit corresponds to a density of $k_{F}^{2} / \pi \simeq 3.2 \times 10^{11} \mathrm{~cm}^{-2}$ ), one obtains from (41)

$$
\frac{\operatorname{Im} \Sigma_{+}^{\mathrm{PA}}\left(k, \epsilon_{k}\right)}{\operatorname{Im} \Sigma_{+}^{\mathrm{DA}}\left(k, \epsilon_{k}\right)} \simeq \frac{20}{\left[k_{F}\right]^{2}} \lambda \bar{\varepsilon}_{0}^{2} \frac{\epsilon_{k}}{1 \mathrm{meV}}
$$

for $k_{B} T \ll \epsilon_{k} \ll \hbar v_{s} k_{\mathrm{TF}}$

Likewise, at nonzero temperatures $\left(\epsilon_{k} / k_{B} \ll T \ll T_{\mathrm{BG}}\right)$, we have from (47)

$$
\frac{\operatorname{Im} \Sigma_{+}^{\mathrm{PA}}\left(k, \epsilon_{k}\right)}{\operatorname{Im} \Sigma_{+}^{\mathrm{DA}}\left(k, \epsilon_{k}\right)} \simeq \frac{100}{\left[k_{F}\right]^{2}} \lambda \bar{\varepsilon}_{0}^{2} \frac{k_{B} T}{1 \mathrm{meV}} .
$$

Finally, at high temperatures $\left(\epsilon_{k} / k_{B} \ll T_{\mathrm{BG}} \ll T\right)$, one obtains from (49) the $k$-independent ratio

$$
\frac{\operatorname{Im} \Sigma_{+}^{\mathrm{PA}}\left(k, \epsilon_{k}\right)}{\operatorname{Im} \Sigma_{+}^{\mathrm{DA}}\left(k, \epsilon_{k}\right)} \simeq \frac{35 g\left(\frac{2 \alpha_{\mathrm{fs}}}{\bar{\varepsilon}_{0}}\right)}{\left[k_{F}\right]} \lambda .
$$

From these ratios we conclude that piezoelectric acoustic phonons can dominate over deformation acoustic phonons in an appreciable range of realistic material parameters, especially for small carrier concentrations. The smaller value of $D \simeq 6.8 \mathrm{eV}$ also found in the literature $[42,43]$ would further increase the relative importance of piezoelectric phonons against intrinsic ones. 


\section{Real part: Anisotropic Fermi velocity}

For the real part of the self-energy, we have, from Eq. (33),

$$
\begin{aligned}
\operatorname{Re} \Sigma_{+}^{\mathrm{PA}}(\mathbf{k}, \omega)= & \sum_{s= \pm} \int \frac{d \mathbf{q}}{(2 \pi)^{2}}\left|\frac{\gamma_{\mathbf{q}}^{\mathrm{PA}}}{\varepsilon_{\mathrm{RPA}}(\mathbf{q}, 0)}\right|^{2} F_{+s}\left(\mathbf{k}, \mathbf{k}^{\prime}\right) \\
& \times\left[\frac{n_{B}\left(\hbar \omega_{\mathbf{q}}\right)+n_{F}\left(\epsilon_{k^{\prime} s}\right)}{\hbar \omega+\hbar \omega_{\mathbf{q}}-\epsilon_{k^{\prime} s}}\right. \\
& \left.+\frac{n_{B}\left(\hbar \omega_{\mathbf{q}}\right)+1-n_{F}\left(\epsilon_{k^{\prime} s}\right)}{\hbar \omega-\hbar \omega_{\mathbf{q}}-\epsilon_{k^{\prime} s}}\right],
\end{aligned}
$$

where the denominators are to be understood as principal values. Unlike for many-body effects directly caused by the electron-electron interaction, this phonon contribution to the electron self-energy tends to be negligibly small compared to the Fermi energy. However, its derivatives are large. As a result, the phonon-induced contributions to the Fermi velocity renormalization are larger than those stemming from the direct electron-electron interactions.

Since $\partial \operatorname{Re} \Sigma_{+}^{\mathrm{PA}}(\mathbf{k}, \omega) / \partial\left(v_{F} \mathbf{k}\right)$ is, by a factor of $v_{s} / v_{F}$, smaller than $\partial \operatorname{Re} \Sigma_{+}^{\mathrm{PA}}(\mathbf{k}, \omega) / \partial \omega$ (see Ref. [44]), it suffices to focus on the frequency derivative, in contrast to the case of electron-electron interactions, where both derivatives matter $[27,45]$. We thus approximate

$$
\tilde{v}_{F}(\hat{\mathbf{k}})=v_{F}\left[1-\left.\frac{\partial \operatorname{Re} \Sigma_{+}^{\mathrm{PA}}\left(\hat{\mathbf{k}} k_{F}, \omega\right)}{\partial \omega}\right|_{\omega=0}\right]^{-1}
$$

for the (direction-dependent) renormalization of the Fermi velocity in graphene induced by piezoelectric acoustic substrate phonons.

For further analysis, it us useful to separate Eq. (33) into three terms,

$$
\Sigma_{+}^{\mathrm{PA}}=\Sigma_{+}^{(\mathrm{ph})}+\Sigma_{+}^{(\mathrm{el})}+\Sigma_{+}^{(\mathrm{vac})}
$$

where $\Sigma_{+}^{(\mathrm{ph})}$ contains just the Bose factor $n_{B}\left(\hbar \omega_{\mathbf{q}}\right), \Sigma_{+}^{(\mathrm{el})}$ contains the Fermi factor $n_{F}\left(\epsilon_{k^{\prime} s}\right)$, and $\Sigma_{+}^{(\mathrm{vac})}$ contains the remaining vacuum term. As in the previous subsection, angleindependent material parameters are assumed in the following.

The real part of $\Sigma_{+}^{(\mathrm{vac})}$ at $\omega=0$ is independent of the Fermi energy:

$$
\operatorname{Re} \Sigma_{+}^{(\mathrm{vac})}(k, 0) \simeq-\frac{\lambda}{16} \frac{v_{s}}{v_{F}}\left[\hbar v_{F} k_{c}+\hbar v_{F} k \log \left(\frac{k_{c}-k}{k}\right)\right],
$$

where $k_{c}$ is a cutoff momentum of the order of the inverse lattice spacing. Because of the small prefactor, $\operatorname{Re} \Sigma_{+}^{(\mathrm{vac})}\left(k_{F}, 0\right)$ represents a weak correction to the chemical potential for all relevant carrier densities, even for $k_{c} \gg k_{F}$. We will see that its derivative can also be neglected because $\partial_{\omega} \operatorname{Re} \Sigma_{+}^{(\mathrm{vac})}\left(k_{F}, 0\right) \simeq$ $(\lambda / 4)\left(v_{s} / v_{F}\right)\left(1+\log k_{F} / k_{c}\right) \ll \partial_{\omega} \operatorname{Re} \Sigma_{+}^{(\mathrm{el})}\left(k_{F}, 0\right)$.

At temperatures $T \ll T_{\mathrm{BG}}$, the term containing the Bose factors $\operatorname{Re} \Sigma_{+}^{(\mathrm{ph})}$ is exponentially small, while at temperatures $T \gg T_{\mathrm{BG}}$ it does not grow larger than a factor $T / T_{\mathrm{BG}}$ times the expression in Eq. (58). Hence we can also neglect $\partial_{\omega} \operatorname{Re} \Sigma_{+}^{(\mathrm{ph})}$.

Thus the only term that can affect the electronic properties is $\operatorname{Re} \Sigma_{+}^{(\mathrm{el})}(\mathbf{k}, \omega)$, which is likewise small in magnitude, at most twice the term shown in Eq. (58), but it has a large derivative.
Note that here the quasielastic approximation $\left(\hbar \omega_{\mathbf{q}} \ll \epsilon_{k^{\prime}}\right)$ is not informative, since $\operatorname{Re} \Sigma_{+}^{(\mathrm{el})}(\mathbf{k}, \omega)$ vanishes when $\hbar \omega_{\mathbf{q}}$ is set to zero.

The integral

$$
\begin{aligned}
& \left.\frac{\partial \operatorname{Re} \Sigma_{+}^{(\mathrm{el})}\left(\hat{\mathbf{k}} k_{F}, \omega\right)}{\partial \omega}\right|_{\omega=0} \\
& =-\sum_{s= \pm} \int \frac{d \mathbf{q}}{(2 \pi)^{2}}\left|\frac{\gamma_{\mathbf{q}}^{\mathrm{PA}}}{\varepsilon_{\mathrm{RPA}}}\right|^{2} F_{+s}\left(\mathbf{k}, \mathbf{k}^{\prime}\right) \\
& \quad \times n_{F}\left(\epsilon_{k^{\prime} s}\right)\left[\frac{1}{\left(\epsilon_{k^{\prime} s}-\hbar \omega_{\mathbf{q}}\right)^{2}}-\frac{1}{\left(\epsilon_{k^{\prime} s}+\hbar \omega_{\mathbf{q}}\right)^{2}}\right]
\end{aligned}
$$

can be computed by changing variables $\left(d \mathbf{q} \rightarrow d \mathbf{k}^{\prime}\right.$, with $\mathbf{k}^{\prime}=$ $\mathbf{k}+\mathbf{q}$ ) and performing the radial integral first by parts, with

$$
u=k^{\prime} n_{F}\left(\epsilon_{k^{\prime} s}\right), \quad d v=\frac{d k^{\prime}}{\left(\epsilon_{k^{\prime} s} \pm \hbar v_{s} q\right)^{2}} .
$$

We arrive at a direction-dependent expression that integrates over the Fermi surface:

$$
\begin{aligned}
\left.\frac{\partial \operatorname{Re} \Sigma_{+}^{(\mathrm{el})}\left(\hat{\mathbf{k}} k_{F}, \omega\right)}{\partial \omega}\right|_{\omega=0}= & -\int_{0}^{2 \pi} \frac{d \alpha}{\hbar v_{F}(2 \pi)^{2}}\left|\frac{\gamma_{\mathbf{k}+\mathbf{q}}^{\mathrm{PA}}}{\varepsilon_{\mathrm{RPA}}}\right|^{2} \\
& \times F_{+s}(\mathbf{k}, \mathbf{k}+\mathbf{q}) \frac{2}{\hbar \omega_{\mathbf{q}}}
\end{aligned}
$$

where, as in (29), $\alpha$ is the angle between $\mathbf{k}$ and $\mathbf{k}+\mathbf{q}$.

After further averaging over the Fermi surface $(\hat{\mathbf{k}}$ directions), the ratio (56) becomes similar to the temperature prefactor of the high-temperature damping (49),

$$
\tilde{v}_{F}=\frac{v_{F}}{1+\frac{\lambda}{4 \pi} f\left(\frac{k_{\mathrm{TF}}}{2 k_{F}}\right)},
$$

where, we recall, all variables are angle-averaged. Inspection of Eq. (61) shows that the renormalization of the Fermi velocity cannot exceed $3 \%$ even for $K_{R}^{2} \sim 1$, and $K_{R}$ is usually much smaller. The result shown in Eq. (61) permits us to confirm the validity of neglecting the vacuum and phonon self-energy parts. A more accurate estimate of the ratios between derivatives yields $\partial_{\omega} \operatorname{Re} \Sigma_{+}^{(\mathrm{vac})} / \partial_{\omega} \operatorname{Re} \Sigma_{+}^{(\mathrm{el})}=$ $O\left(v_{s} / v_{F}\right) \ll 1$, while $\partial_{\omega} \operatorname{Re} \Sigma_{+}^{(\mathrm{ph})} / \partial_{\omega} \operatorname{Re} \Sigma_{+}^{(\mathrm{el})}$ is $O\left(v_{s} / v_{F}\right)$ for $T \ll T_{\mathrm{BG}}$ and $O\left(T / T_{F}\right)$ for $T_{\mathrm{BG}} \ll T \ll T_{F}$.

\section{Electron mobility \\ 1. Transport lifetime}

Within Boltzmann transport theory, the momentum (or transport) relaxation time $\tau_{+\operatorname{tr}}(\mathbf{k})$ (where the subscript denotes "transport" and + denotes the band) is calculated analogously to the inverse lifetime in Sec. IV B, but with an extra angular factor $(1-\cos \alpha)=q^{2} / 2 k^{2}$ in the integrand, which increases the weight of large-angle scattering processes. Specifically, Eq. (37) is replaced by

$$
\begin{aligned}
\frac{\hbar}{2 \tau_{+\mathrm{tr}}^{\mathrm{PA}}(\mathbf{k})}= & \pi \sum_{s= \pm} \sum_{t= \pm} \int \frac{d \mathbf{q}}{(2 \pi)^{2}} \frac{q^{2}}{2 k^{2}}\left|\frac{\gamma_{\mathbf{q}}^{\mathrm{PA}}}{\varepsilon_{\mathrm{RPA}}(\mathbf{q}, 0)}\right|^{2} \frac{1+s \cos \alpha}{2} \\
& \times\left[n_{F}\left(\hbar \omega_{\mathbf{q}}+t \epsilon_{k}\right)+n_{B}\left(\hbar \omega_{\mathbf{q}}\right)\right] \delta\left(\epsilon_{k+}-\epsilon_{k^{\prime} s}\right)
\end{aligned}
$$


where the quasielastic approximation has been made. The inclusion of this additional $q^{2}$ factor in the integrand improves the quasielastic approximation, changes the power-law scaling at low temperatures (by generating an extra factor $T^{2}$ ), and corrects the lifetime with a constant factor at temperatures greater than $T_{\mathrm{BG}}$.

\section{Low temperature}

For quasiparticle energies such that $\left(\epsilon_{k} / k_{B} \ll T\right)$, we find (after angle averaging) results that are essentially independent of $\epsilon_{k}$, i.e., $\tau_{+\mathrm{tr}}^{\mathrm{PA}}(k) \simeq \tau_{+\mathrm{tr}}^{\mathrm{PA}}\left(k_{F}\right)$. In the low, yet nonzero, temperature regime $\epsilon_{k} / k_{B} \ll T \ll 2 r_{s} T_{\mathrm{BG}}$, we obtain

$$
\begin{aligned}
\frac{\hbar}{2 \tau_{+\mathrm{tr}}^{\mathrm{PA}}\left(k_{F}\right)} & \simeq \frac{\lambda k_{B} T}{8}\left(\frac{k_{B} T}{\hbar v_{s} k_{\mathrm{TF}}}\right)^{4} \frac{k_{\mathrm{TF}}^{2}}{k_{F}^{2}} \int_{0}^{\infty} d x x^{4} \operatorname{csch}(x) \\
& =\frac{\lambda k_{B} T}{8}\left(\frac{\bar{\varepsilon}_{0}}{\alpha_{\mathrm{fs}}}\right)^{2}\left(\frac{T}{T_{\mathrm{BG}}}\right)^{4} \frac{93 \zeta(5)}{2}
\end{aligned}
$$

$[93 \zeta(5) / 2 \simeq 48.2]$, which should be compared to Eq. (47). The shift from $T^{3}$ to $T^{5}$ behavior is due to the transport-induced reduced weight (by a factor $q^{2} / 2 k_{F}^{2}$ ) of the low- $q$ values dominating the inverse transport lifetime at low temperatures.

If the vertex screening is neglected, we still obtain a convergent result, despite the temperature being nonzero, because the low- $q$ divergence is already suppressed by the transport-associated angular weighting factor. We obtain

$$
\frac{\hbar}{2 \tau_{+\mathrm{tr}}^{\mathrm{PA}}\left(k_{F}\right)_{(\mathrm{no} \mathrm{scr})}} \simeq \frac{\lambda 7 \zeta(3)}{4} k_{B} T\left(\frac{T}{T_{\mathrm{BG}}}\right)^{2},
$$

and we recall that the nontransport equivalent of this equation is divergent, as discussed in Sec. IV B [see discussion before (47)]. The limit (64) is coincident with the $T^{3}$ dependence found in Ref. [43], where vertex screening in the particular case of GaAs is not taken into account. The neglect of vertex screening is acceptable in the temperature regime $2 r_{s} T_{\mathrm{BG}} \lesssim$ $T \ll T_{\mathrm{BG}}$ in those materials with $4 r_{s} \ll 1$, because in that case the integral in Eq. (62) is dominated by exchanged momenta $q$ such that $k_{\mathrm{TF}} \ll q \ll k_{F}$, which are a little bit sensitive to vertex screening. This intermediate regime of temperatures does not exist for substrate materials such that $2 r_{s} \sim 1$.

\section{High temperature}

For the high-temperature range $T \gg T_{\mathrm{BG}}$, we have

$$
\frac{\hbar}{2 \tau_{+\mathrm{tr}}^{\mathrm{PA}}\left(k_{F}\right)} \simeq \frac{\lambda}{2} k_{B} T \int_{0}^{1} x \sqrt{1-x^{2}} d x=\frac{\lambda}{6} k_{B} T,
$$

to be compared with Eq. (49). The absence of a qualitative change in the temperature dependence as we shift from nontransport to transport lifetime is due to the relatively small weight, at high temperatures, of the transport-reduced, low- $q$ processes.

Thus we see that the transport scattering rates are comparable to the previous imaginary self-energies except for an extra $\left(T / T_{\mathrm{BG}}\right)^{2}$ factor appearing at low temperatures due to extra angular suppression of the otherwise dominant low- $q$ events. A similar comparison holds for the intrinsic acoustic deformation-potential phonons, where

$$
\frac{\hbar}{2} \tau_{+\mathrm{tr}}^{\mathrm{DA}}\left(k_{F}\right)^{-1} \simeq 10\left(\frac{T}{T_{\mathrm{BG}}}\right)^{2} \operatorname{Im} \Sigma_{+}^{\mathrm{DA}}\left(k_{F}, 0\right)
$$

at low temperatures, while

$$
\frac{\hbar}{2} \tau_{+\mathrm{tr}}^{\mathrm{DA}}\left(k_{F}\right)^{-1} \simeq \frac{1}{2} \operatorname{Im} \Sigma_{+}^{\mathrm{DA}}\left(k_{F}, 0\right)
$$

for high temperatures. In the last two equations, we are comparing the results of Refs. [43] and [26] for the transport scattering rate and the inverse lifetime, respectively.

\section{Comparison with graphene intrinsic phonons}

In analogy with Sec. IV B, we may compare the transport rates due to deformation and piezoelectric phononic modes. In the low-temperature limit (as before, $\left[k_{F}\right]$ is $k_{F}$ in units of $10^{6} \mathrm{~cm}^{-1}$ ),

$$
\frac{\tau_{+\mathrm{tr}}^{\mathrm{PA}}\left(k_{F}\right)^{-1}}{\tau_{+\mathrm{tr}}^{\mathrm{DA}}\left(k_{F}\right)^{-1}} \simeq \frac{200}{\left[k_{F}\right]^{2}} \lambda \bar{\varepsilon}_{0}^{2} \frac{k_{B} T}{1 \mathrm{meV}},
$$

while at temperatures above $T_{\mathrm{BG}}$,

$$
\frac{\tau_{+\mathrm{tr}}^{\mathrm{PA}}\left(k_{F}\right)^{-1}}{\tau_{+\mathrm{tr}}^{\mathrm{DA}}\left(k_{F}\right)^{-1}} \simeq \frac{45}{\left[k_{F}\right]} \lambda,
$$

independent of temperature. Upon inserting the specific material parameters, Eq. (69) is in agreement with the calculations of Ref. [43], where PA and DA transport rates are compared for GaAs. Equations (68) and (69) must be compared to Eqs. (53) and (54) of Sec. IV B, respectively. As in the nontransport lifetime estimates presented therein, we note that piezoelectric phonons dominate over deformation phonons at nonsmall couplings and low densities. We recall that Ref. [43] used a deformation constant $D=6.8 \mathrm{eV}$, quite smaller than the value $D=25 \mathrm{eV}$ [26] we have used here. That replacement reduces $1 / \tau^{\mathrm{DA}}$ by about a factor of 10 and makes the substrate PA phonons relatively more important.

\section{Mobility}

Finally, in order to compute the electron mobility, we average the momentum relaxation time [see Eq. (62)],

$$
\overline{\tau_{\text {tr }}} \equiv \int d \epsilon D(\epsilon) \tau_{+\operatorname{tr}}(k(\epsilon))\left[-d n_{F}(\epsilon) / d \epsilon\right],
$$

and because the energy derivative peaks at $E_{F}$ while $\tau_{+\operatorname{tr}}(k)$ varies slowly with $k$, one can write the classical Drude formula for the mobility,

$$
\mu=\frac{e \tau_{+\operatorname{tr}}\left(k_{F}\right)}{m^{*}}
$$

in terms of $\tau_{+ \text {tr }}(k)$ computed at the Fermi level and the "effective mass" $m^{*}=\hbar k_{F} / v_{F}$ of the graphene Dirac fermions.

\section{NUMERICAL RESULTS}

In the following, we present and discuss numerical results for the various rates and mean free paths derived in Secs. IV B and IV D. Unless otherwise stated, the numerical values of this section are computed for $\mathrm{ZnO}$ substrates $(Z$ cut), which are isotropic (see Fig. 2) and whose parameters are $\lambda=0.03$ 

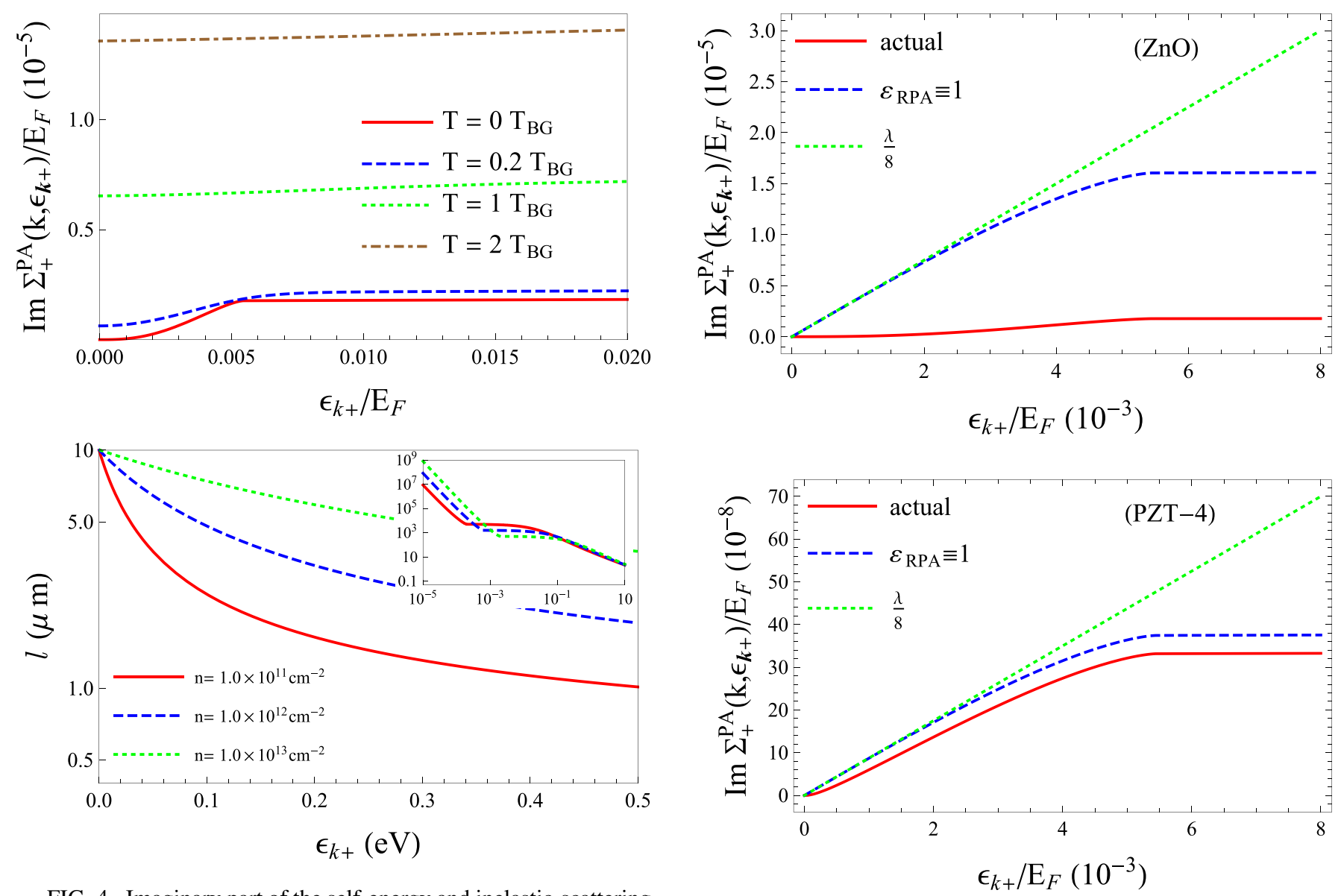

FIG. 4. Imaginary part of the self-energy and inelastic-scattering length of charge carriers in graphene on $\mathrm{ZnO}(Z$ cut) as a function of the energy, $\epsilon_{k} / E_{F}=\left(k-k_{F}\right) / k_{F}$. Upper: $\operatorname{Im} \Sigma_{+}^{\mathrm{PA}}$ for different temperatures. The curves are valid for all densities. Lower: inelastic mean free path $l$ for different carrier concentrations at room temperature $\left(T=300 \mathrm{~K}=26 \mathrm{meV} / k_{B}\right)$. The inset shows $l$ at $T=0$ for the same densities. The Bloch-Grüneisen temperature $T_{\mathrm{BG}}$ is given in Eq. (40). For this material, $k_{B} T_{\mathrm{BG}}=0.0054 E_{F}$ (for all carrier densities) and $k_{\mathrm{TF}} / k_{\mathrm{F}} \simeq 2$ (thus $T_{\mathrm{BG}} \simeq \hbar v_{s} k_{\mathrm{TF}} / k_{B}$ ). For these three densities, $k_{B} T_{\mathrm{BG}}=0.2,0.63$, and $2 \mathrm{meV}$, while $E_{F}=37.4,117$, and $374 \mathrm{meV}$.

and $\bar{\varepsilon}_{0}=4.8$, which implies $k_{\mathrm{TF}} / k_{F} \simeq 2$ and $k_{B} T_{\mathrm{BG}} / E_{F} \simeq$ 0.0054 . Estimates for other substrate material parameters can be readily obtained from inspection of the various analytical limiting expressions derived in the previous section.

\section{A. Imaginary part}

In the upper plot of Fig. 4, we show the imaginary part of the on-shell self-energy as a function of the parameter $\epsilon_{k} / E_{F}>0$ for different temperatures. The curves are universal in the sense that they are density-independent. The zero-temperature curve shows, for small $\epsilon_{k}$, the limiting $\epsilon_{k}^{3}$ behavior of Eq. (41), which arises due to the combined effect of screening and the phasespace restrictions faced by the electrons when losing energy via phonon emission. This restriction disappears when $\epsilon_{k}$ is greater than any phononic energy, i.e., $\epsilon_{k} \gg k_{B} T_{\mathrm{BG}}$. Above this threshold, the imaginary part of the self-energy becomes energy-independent, as predicted by Eq. (44). At still higher

FIG. 5. Low-energy behavior of the imaginary part of the selfenergy at zero temperature. The solid, dashed, and dotted lines correspond, respectively, to the exact values, the values without vertex screening, and the values obtained (in the unscreened case) from the linear $\lambda / 8$ approximation of Eq. (46). Upper: graphene on $\mathrm{ZnO}(Z$ cut). Lower: graphene on PZT-4 ( $Z$ cut), for which $k_{B} T_{\mathrm{BG}} / E_{F}=$ $0.0045, k_{\mathrm{TF}} / k_{\mathrm{F}} \simeq 0.025$, and $\hbar v_{s} k_{\mathrm{TF}} / E_{F} \simeq 5.7 \times 10^{-5}$. See Table I for $\lambda$ values.

energies $\left(\epsilon_{k} \gg E_{F}\right.$, not shown in the upper plot of Fig. 4), it increases linearly with the length of the constant energy circumference at the quasiparticle energy $E_{k+} \propto k$. Such a linear increase with $k$ would appear with a negligible slope in the tiny scale of $\epsilon_{k} \propto\left(k-k_{F}\right)$ of the upper plot of Fig. 4. Specifically, the slope is, in the dimensionless units of the upper plot of Fig. 4, $(\lambda \pi / 16)\left(v_{s} / v_{F}\right)$.

The upper plot of Fig. 4 also shows that a further increase in temperature $\left(T>T_{\mathrm{BG}}\right.$ ) smears these features due to phonon excitation and electron heating near the Fermi energy, as exemplified in Eq. (49). The effect of vertex screening in the regime of low $\epsilon_{k}$ and low $T$ can be appreciated in Fig. 5 for both $\mathrm{ZnO}$ and (angle-averaged) PZT substrates with its higher dielectric constant (and thus smaller $\lambda$ ). For the sake of comparison, the graphics include also the linear approximation (46), which holds better for PZT because its large dielectric constant reduces the size of the phase-space region where the screening of the phonon interaction by the electron cloud (vertex correction) is really important. Unlike for $\mathrm{ZnO}$, in this material $k_{\mathrm{TF}}$ is considerably smaller than $k_{F}$, 

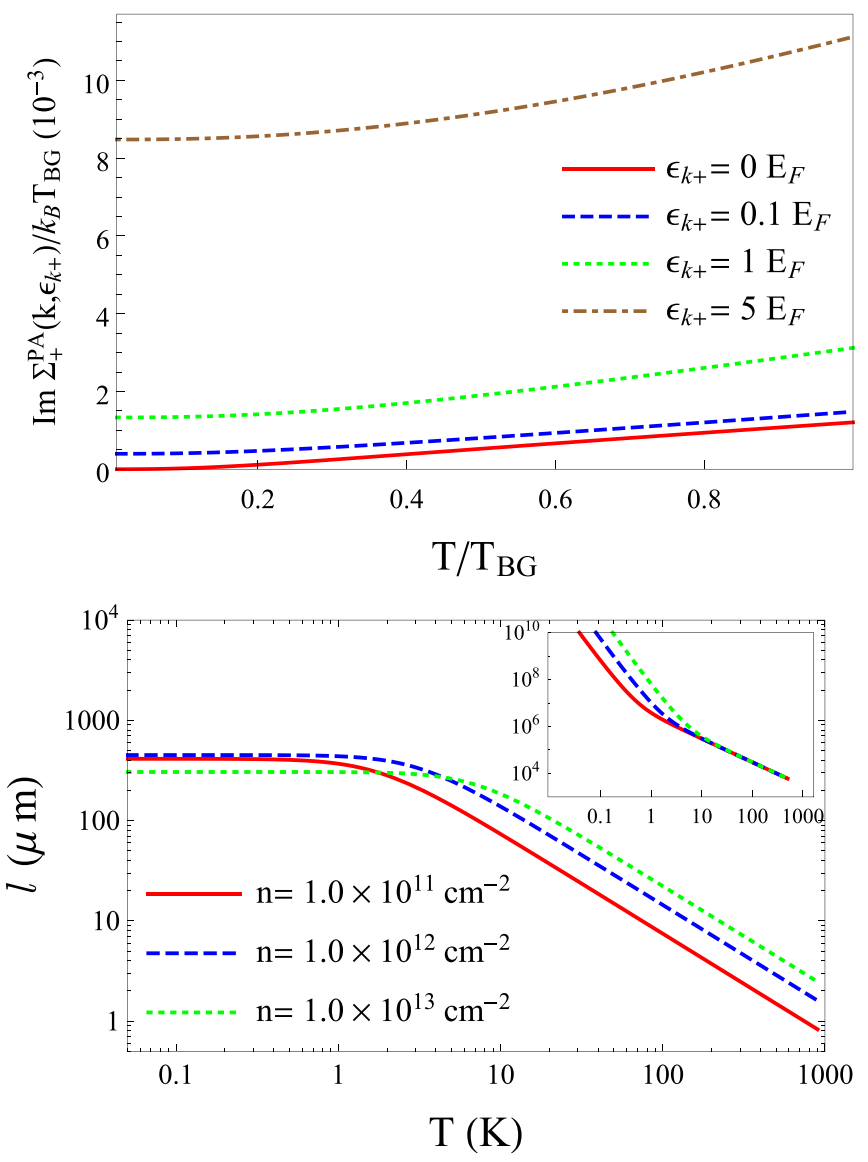

FIG. 6. Imaginary part of the self-energy and inelastic-scattering length. Upper: $\operatorname{Im} \Sigma_{+}^{\mathrm{PA}}$ as a function of $T / T_{\mathrm{BG}}$ for different values of $\epsilon_{k} / E_{F}$. Lower: $l$ as a function of $T$ for $\epsilon_{k}=0.1 \mathrm{eV} \simeq 1160 \mathrm{~K}$ and different doping levels. The inset shows the corresponding curves for $\epsilon_{k}=0$. Here, $E_{F} \simeq 185 k_{B} T_{\mathrm{BG}}$. The values of $T_{\mathrm{BG}}$ for these three densities are $2.35,7.35$, and $23.5 \mathrm{~K}$.

which leaves room for an intermediate range of $\epsilon_{k}$ values for which the approximation $\varepsilon_{\mathrm{RPA}} \simeq 1$ is acceptable while the linear behavior still holds. As announced in Sec. IV B, after Eq. (46), there is an offset between the true imaginary self-energy and the linear approximation due to the reduced contribution of the screened low- $q$ processes.

The upper plot of Fig. 6 shows the temperature dependence of $\operatorname{Im} \Sigma_{+}^{\mathrm{PA}}$ for fixed values of $\epsilon_{k}$. At low temperatures $\left(T \ll \epsilon_{k}\right.$, hot electron regime), these decay linewidths are independent of $T$. Note that in this figure the nonzero values of $\epsilon_{k}$ are well above $\hbar v_{s} k_{\mathrm{TF}}$ and thus the limit (41) does not apply. At higher temperatures ( $T>T_{\mathrm{BG}}$ ), the linear behavior of Eq. (49) is recovered.

\section{B. Inelastic mean free path}

The lower plots of Figs. 4 and 6 are devoted to the inelasticscattering mean free path, which is the inverse of the imaginary part of the on-shell self-energy:

$$
l(k)=\frac{\hbar v_{F}}{2 \operatorname{Im} \Sigma_{+}^{\mathrm{PA}}\left(k, \epsilon_{k}\right)} .
$$

The lower plot of Fig. 4 shows values for $l(k)$ as a function of $\epsilon_{k}$ for three cases of typical doping conditions. Note that they tend to coincide at small $\epsilon_{k}$, as suggested by Eq. (49) (case $\left.T>T_{\mathrm{BG}}\right)$, which predicts a doping-independent low- $\epsilon_{k}(k \rightarrow$ $k_{F}$ ) limit at nonzero temperatures. Finally, the inset of the lower plot of Fig. 4 clearly displays the three energy regimes that hold at zero temperature and which can be inferred from Eqs. (41)-(45).

The temperature dependence of $l$ is shown in the lower plot of Fig. 6. A crossover from ( $T$-independent) low-temperature to $\left(T^{-1}\right)$ high-temperature behavior can be appreciated for $T \sim T_{\mathrm{BG}}$, in agreement with Eqs. (41) and (49). One must note, however, that Eq. (41) does not truly apply to the lowtemperature sector of this graph, because here $\epsilon_{k}>\hbar v_{s} k_{\mathrm{TF}}$, unlike the assumption in (41). This explains the discrepancy in the density dependence. For this material, $\hbar v_{s} k_{\mathrm{TF}}$ takes values $0.2,0.63$, and $2 \mathrm{meV}$ for the three listed densities, all much smaller than the value $\epsilon_{k}=100 \mathrm{meV}$ considered there.

The inset shows the corresponding curves for $\epsilon_{k}=0$. A clear crossover for $T^{-3}$ to $T^{-1}$ behavior is observed at $T \sim$ $T_{\mathrm{BG}}$, in agreement with Eqs. (47) and (49).

\section{Density dependence}

For a fixed value of $k$ and at room temperature, Fig. 7 shows the variation of $\operatorname{Im} \Sigma_{+}^{\mathrm{PA}}$ and of the mean free path as a
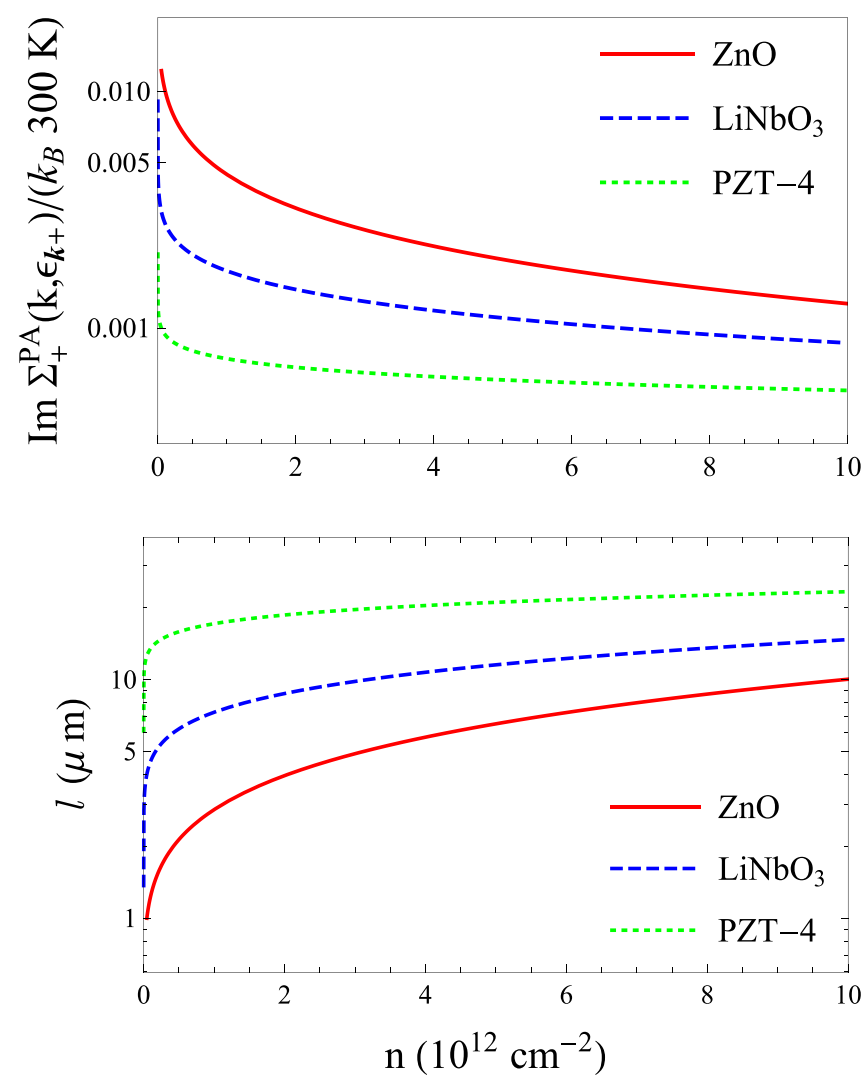

FIG. 7. Imaginary part of the self-energy and inelastic-scattering length as a function of doping, for different materials, at fixed (room) temperature and electronic state $k=\sqrt{\pi \times 10^{13} \mathrm{~cm}^{-2}}$ (recall $k_{F}=$ $\sqrt{\pi n}$ ). Upper: $\operatorname{Im} \Sigma_{+}^{\mathrm{PA}}$ as a function of carrier density $n$. Lower: $l$ as a function of $n$ in the same units for the same materials. 

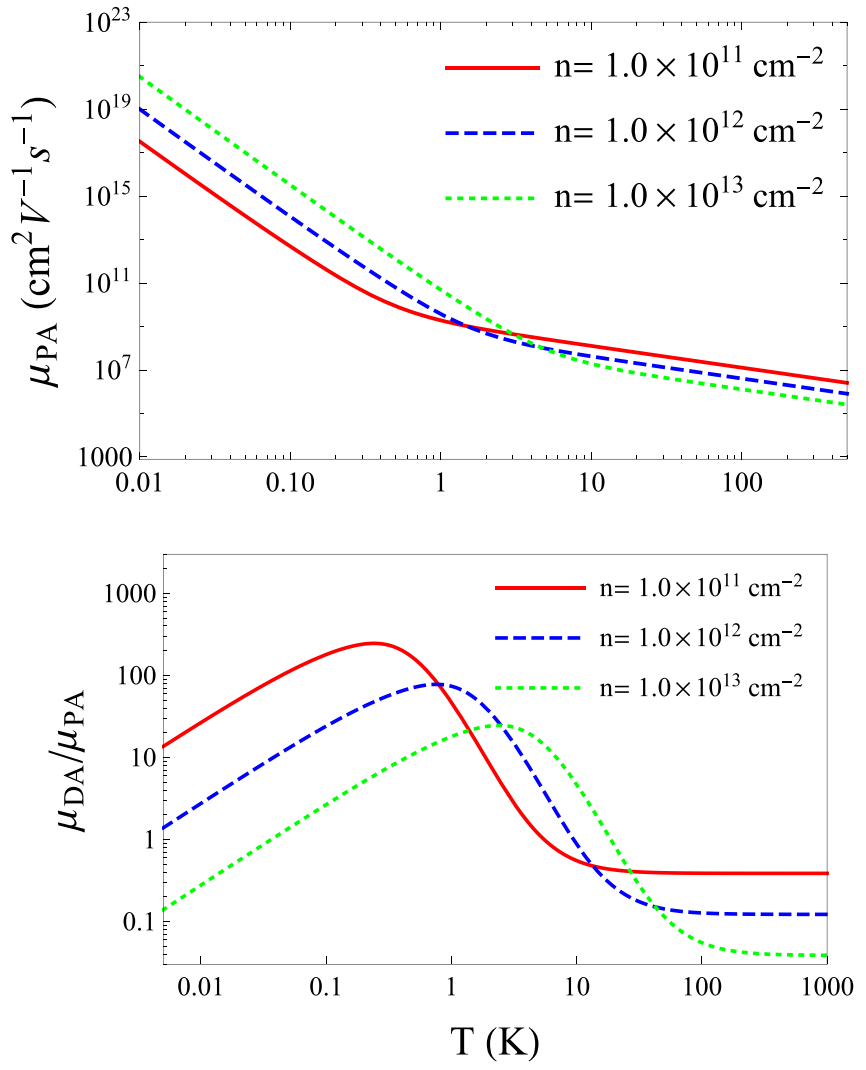

FIG. 8. Electron mobility due only to piezoelectric acoustic phonons and its comparison with that due to intrinsic phonons. Upper: the mobility $\mu_{\mathrm{PA}}$ as a function of the temperature, for several carrier concentrations. Lower: the ratio $\mu_{\mathrm{DA}} / \mu_{\mathrm{PA}}$, where $\mu_{\mathrm{DA}}$ is the mobility obtained when only the deformation potential of intrinsic phonons (with $D=25 \mathrm{eV}$ ) is included. The ratio between mobilities must be increased by a factor $(25 / 6.8)^{2} \simeq 13.5$ when the value $D=6.8 \mathrm{eV}$ is used [43]. The values of $T_{\mathrm{BG}}$ for these doping levels are given in the caption of Fig. 6, while $T_{F}=434,1360$, and $4340 \mathrm{~K}$.

function of the carrier density. A logarithmic divergence in the linewidth, accompanied by a vanishing mean free path, is seen to appear in the undoped regime, where the description of the system employed in the present paper is not valid anymore. This spurious low-doping behavior can be expected from an extrapolation of Eq. (49) to low doping.

\section{Mobility: Comparison with intrinsic phonons}

In the upper plot of Fig. 8, we show the electron mobility $\mu$ [see Eq. (71)] due only to piezoelectric phonons. The $T^{-5}$ and $T^{-1}$ behaviors can be appreciated at low and high temperatures, respectively, as expected from Eqs. (63) and (65), taking into account Eq. (71) for the density dependence.

Finally, in the lower plot of Fig. 8 we compare the substrateinduced mobility to that stemming only from graphene intrinsic phonons, with $D=25 \mathrm{eV}$. The total combined mobility due to (piezoelectric and intrinsic deformation) acoustic phonons is $\mu=\left(\mu_{\mathrm{PA}}^{-1}+\mu_{\mathrm{DA}}^{-1}\right)^{-1}$. Specifically, we plot the ratio between the two inverse mobilities. The smaller value of $D=6.8 \mathrm{eV}$ reduces the intrinsic inverse mobility by an order of magnitude and correspondingly increases the relative importance of piezoelectric phonons. This ratio between transport scattering rates shows two clear low- and high- $T$ regimes with linear-in- $T$ and $T$-independent behaviors, respectively, in agreement with Eqs. (68) and (69). At low and high temperatures, the relative importance of the PA phonons increases with decreasing density. There is an intermediate temperature regime in which the density dependence is inverted. Thus we see that the piezoelectric phonons dominate over a wide range of temperatures and densities. If $D=6.8 \mathrm{eV}$ is chosen for the intrinsic phonons, then the momentum relaxation due to PA phonons computed here prevails essentially always except at very high temperature and density or for extremely low temperatures.

\section{CONCLUSIONS}

We have studied the effective interaction of charge carriers in graphene on a piezoelectric substrate, as modified by the acoustic phonons of the piezoelectric substrate. Our diagrammatic approach takes into account the renormalization of both phonon modes and carrier states due to the mutual interaction, and it emphasizes the importance of all the involved screening processes for a correct evaluation of the mean free path and carrier mobility. We have obtained numerous analytical limits as a function of carrier energy, density, and temperature, which have allowed us to understand the trends shown by the numerical results.

We may emphasize that the inverse mobility due to piezoelectric acoustic phonons increases with carrier density at high temperatures while it is a decreasing function of density at low temperatures (see the upper plot in Fig. 8), the latter trend being understandable in terms of increased electron screening at high densities. On the other hand, the temperature dependence of the inverse mobility is much more pronounced at low temperatures.

When compared with the values obtained when only intrinsic deformation phonons are taken into account, we find that the contributions of the piezoelectric acoustic phonons to the inverse lifetime and mobility dominate over a considerable range of temperatures and doping levels, a parameter range that becomes almost pervasive if low values of the deformation coupling constant are chosen from the literature.

As our results are applicable to piezoelectric materials of various lattice symmetries and interaction strengths, they will be helpful in the development of electronic devices involving graphene deposited on piezoelectric substrates. Among such potential devices, we may mention the graphene field-effect transistor on a piezoelectric substrate as studied experimentally in Refs. [24] and [25] with PZT and $\mathrm{LiNbO}_{3}$, respectively. That class of setups was also investigated as a possible basis for the building of nonvolatile memories [46]. Finally, a suitable understanding of the interaction between graphene electrons and acoustic piezoelectric phonons will enhance applications based on the use of piezoelectric surface acoustic waves beneath a graphene layer.

\section{ACKNOWLEDGMENTS}

We wish to thank Fernando Calle and Jorge Pedrós for valuable discussions. This work has been supported by 
Spain's MINECO through Grants No. FIS2011-23713 and No. FIS2013-41716-P; the European Research Council Advanced Grant (Contract No. 290846), and the European Commission under the Graphene Flagship, Contract No. CNECTICT604391. D.G.G. acknowledges financial support from Campus de Excelencia Internacional (Campus Moncloa UCM-UPM).
[1] R. Weigel, D. Morgan, J. Owens, A. Ballato, K. Lakin, K.-Y. Hashimoto, and C. Ruppel, IEEE Trans. Microw. Theory Tech. 50, 738 (2002).

[2] E. A. Cerda-Méndez, D. Sarkar, D. N. Krizhanovskii, S. S. Gavrilov, K. Biermann, M. S. Skolnick, and P. V. Santos, Phys. Rev. Lett. 111, 146401 (2013).

[3] C. Ruppert, J. Neumann, J. B. Kinzel, H. J. Krenner, A. Wixforth, and M. Betz, Phys. Rev. B 82, 081416 (2010).

[4] J. Schiefele, J. Pedrós, F. Sols, F. Calle, and F. Guinea, Phys. Rev. Lett. 111, 237405 (2013).

[5] P. V. Santos, T. Schumann, M. H. Oliveira, J. M. J. Lopes, and H. Riechert, Appl. Phys. Lett. 102, 221907 (2013).

[6] V. Miseikis, J. E. Cunningham, K. Saeed, R. O'Rorke, and A. G. Davies, Appl. Phys. Lett. 100, 133105 (2012).

[7] L. Bandhu, L. M. Lawton, and G. R. Nash, Appl. Phys. Lett. 103, 133101 (2013).

[8] P. Thalmeier, B. Dóra, and K. Ziegler, Phys. Rev. B 81, 041409 (2010).

[9] A. H. Castro Neto, F. Guinea, N. M. R. Peres, K. S. Novoselov, and A. K. Geim, Rev. Mod. Phys. 81, 109 (2009).

[10] A. C. Ferrari et al., Nanoscale 7, 4598 (2015).

[11] K. Bolotin, K. Sikes, Z. Jiang, M. Klima, G. Fudenberg, J. Hone, P. Kim, and H. Stormer, Solid State Commun. 146, 351 (2008).

[12] R. C. Dean, A. F. Young, I. Meric, C. Lee, L. Wang, S. Sorgenfrei, K. Watanabe, T. Taniguchi, P. Kim, K. L. Shepard, and J. Hone, Nat. Nanotechnol. 5, 722 (2010).

[13] S. Fratini and F. Guinea, Phys. Rev. B 77, 195415 (2008).

[14] J.-H. Chen, C. Jang, S. Xiao, M. Ishigami, and M. S. Fuhrer, Nat. Nanotechnol. 3, 206 (2008).

[15] K. Zou, X. Hong, D. Keefer, and J. Zhu, Phys. Rev. Lett. 105, 126601 (2010).

[16] J. Schiefele, F. Sols, and F. Guinea, Phys. Rev. B 85, 195420 (2012).

[17] B. Amorim, J. Schiefele, F. Sols, and F. Guinea, Phys. Rev. B 86, 125448 (2012).

[18] Z.-Y. Ong and M. V. Fischetti, Phys. Rev. B 86, 165422 (2012).

[19] Z.-Y. Ong and M. V. Fischetti, Appl. Phys. Lett. 102, 183506 (2013).

[20] Z.-Y. Ong and M. V. Fischetti, Phys. Rev. B 88, 045405 (2013).

[21] E. H. Hwang and S. Das Sarma, Phys. Rev. B 87, 115432 (2013).

[22] H. Ezawa, Ann. Phys. (N.Y.) 67, 438 (1971).

[23] W.-K. Tse, E. H. Hwang, and S. Das Sarma, Appl. Phys. Lett. 93, 023128 (2008).
[24] X. Hong, A. Posadas, K. Zou, C. H. Ahn, and J. Zhu, Phys. Rev. Lett. 102, 136808 (2009).

[25] S. Bidmeshkipour, A. Vorobiev, M. A. Andersson, A. Kompany, and J. Stake, Appl. Phys. Lett. 107, 173106 (2015).

[26] Q. Li and S. Das Sarma, Phys. Rev. B 87, 085406 (2013).

[27] G. D. Mahan, Many-Particle Physics (Springer Science \& Business Media, Boston, 2013).

[28] S. H. Simon, Phys. Rev. B 54, 13878 (1996).

[29] D. G. González, F. Sols, F. Guinea, and I. Zapata, Phys. Rev. B 94, 085423 (2016).

[30] B. Wunsch, T. Stauber, F. Sols, and F. Guinea, New J. Phys. 8, 318 (2006).

[31] E. H. Hwang and S. Das Sarma, Phys. Rev. B 75, 205418 (2007).

[32] R. D. Mattuck, A Guide to Feynman Diagrams in the Many-Body Problem (Courier, New York, 2012).

[33] D. Royer and E. Dieulesaint, Elastic Waves in Solids I: Free and Guided Propagation (Springer Science \& Business Media, Berlin, 2000).

[34] B. Auld, Acoustic Fields and Waves in Solids (Krieger, Malabar, 1990).

[35] A. Knäbchen, Y. B. Levinson, and O. Entin-Wohlman, Phys. Rev. B 54, 10696 (1996).

[36] J. Pedrós, L. García-Gancedo, C. J. B. Ford, C. H. W. Barnes, J. P. Griffiths, G. A. C. Jones, and A. J. Flewitt, J. Appl. Phys. 110, 103501 (2011).

[37] A. Wixforth, J. Scriba, M. Wassermeier, J. P. Kotthaus, G. Weimann, and W. Schlapp, Phys. Rev. B 40, 7874 (1989).

[38] J. Campbell and W. Jones, IEEE Trans. Sonics Ultrason. 15, 209 (1968).

[39] R. S. Weis and T. K. Gaylord, Appl. Phys. A 37, 191 (1985).

[40] S. Sanna and W. G. Schmidt, Phys. Rev. B 81, 214116 (2010).

[41] W.-K. Tse and S. Das Sarma, Phys. Rev. Lett. 99, 236802 (2007).

[42] K. Kaasbjerg, K. S. Thygesen, and K. W. Jacobsen, Phys. Rev. B 85, 165440 (2012).

[43] S. H. Zhang, W. Xu, S. M. Badalyan, and F. M. Peeters, Phys. Rev. B 87, 075443 (2013).

[44] A. B. Migdal, Sov. Phys. JETP 34, 996 (1958).

[45] S. Das Sarma, E. H. Hwang, and W.-K. Tse, Phys. Rev. B 75, 121406 (2007).

[46] E. B. Song, B. Lian, S. M. Kim, S. Lee, T.-K. Chung, M. Wang, C. Zeng, G. Xu, K. Wong, Y. Zhou, H. I. Rasool, D. H. Seo, H.-J. Chung, J. Heo, S. Seo, and K. L. Wang, Appl. Phys. Lett. 99, 042109 (2011) 